TITLE:

\title{
Numerical simulation using a Hamiltonian particle method for effective elastic properties in cracked media
}

\section{$\operatorname{AUTHOR}(\mathrm{S}):$}

Takekawa, Junichi; Mikada, Hitoshi; Goto, Tadanori

\section{CITATION:}

Takekawa, Junichi ... [et al]. Numerical simulation using a Hamiltonian particle method for effective elastic properties in cracked media. Exploration Geophysics 2014, 45(2): 116-124

\section{ISSUE DATE:}

2014-01-21

URL:

http://hdl.handle.net/2433/188919

\section{RIGHT:}

(C) CSIRO 2014; This is not the published version. Please cite only the published version.; この論文は出版社版でありません。引用の際には 出版社版をご確認ご利用ください。 


\section{NUMERICAL SIMULATION USING A HAMILTONIAN PARTICLE METHOD \\ FOR EFFECTIVE ELASTIC PROPERTIES IN CRACKED MEDIA}

Junichi Takekawa

C1-1-111, Kyotodaigaku-Katsura, Nishikyo-ku, Kyoto

Japan, 615-8540

Phone: $+81-75-383-3197$

Facsimile: +81-75-383-3198

E-mail: takekawa@tansa.kumst.kyoto-u.ac.jp

Hitoshi Mikada

C1-1-112, Kyotodaigaku-Katsura, Nishikyo-ku, Kyoto

Japan, 615-8540

Phone: +81-75-383-3196

Facsimile: $+81-75-383-3198$

E-mail:mikada@gakushikai.jp

Tada-nori Goto

C1-1-113, Kyotodaigaku-Katsura, Nishikyo-ku, Kyoto

Japan, 615-8540

Phone: +81-75-383-3195

Facsimile: $+81-75-383-3198$

E-mail: goto.tadanori.8a@kyoto-u.ac.jp

Left Running Heading: J. Takekawa et al.

Right Running Heading: A particle method for cracked media 


\begin{abstract}
We apply a Hamiltonian particle method, which is one of the particle methods, to simulate seismic wave propagation in a cracked medium. In a particle method, traction-free boundaries can readily be implemented and the spatial resolution can be chosen in an arbitrary manner. Utilisation of the method enables us to simulate seismic wave propagation in a cracked medium and to estimate effective elastic properties derived from the wave phenomena. These features of the particle method would bring some advantages of numerical efficiencies (e.g. calculation time, computational memory) and the reduction of time for pre-processing.
\end{abstract}

We first describe our strategy for the introduction of free surfaces inside a rock mass, i.e., cracks, and to refine the spatial resolution in an efficient way. We then model a 2D cracked medium which contains randomly distributed, randomly oriented, rectilinear, dry and nonintersecting cracks, and simulate the seismic wave propagation of P- and SV-plane waves through the region. We change the crack density in the cracked region and determine the effective velocity in the region. Our results show good agreement with the modified selfconsistent theory, which is one of the effective-medium theories. Finally, we investigate the influence of the ratio of crack length to particle spacing on the calculated effective velocities. The effective velocity obtained becomes almost constant when the ratio of crack length to particle spacing is more than approximately 20. Based on this result, we propose to use more than 20 particles per crack length.

Key Words: particle method, effective elastic property, cracked media, digital rock physics. 


\section{INTRODUCTION}

It is important to estimate physical properties of cracked rocks in many scientific and engineering fields. Especially in exploration geophysics, understanding the behaviour of seismic waves in cracked rock masses is of interest when prospecting for earth resources. In the past, many theoretical studies have been directed at estimating the elastic properties of cracked media. O'Connell and Budiansky (1974) proposed a model for the macroscopic elastic properties based on a self-consistent approximation. Budiansky and O'Connell (1976) calculated the elastic moduli of bodies, in which planar cracks are randomly distributed, on the basis of the self-consistent method. Henyey and Pomphrey (1982) proposed a modified self-consistent theory with a set of differential equations for the effective elastic moduli, taking into account the crack interaction energy. Davis and Knopoff (1995) showed that the mean-field approximation, also known as the theory for noninteracting cracks, is appropriate for the problem of finding the modulus of a material with a high concentration of cracks by using a boundary integral method. In spite of a variety of approaches, theoretical approaches suffer from limitations such as crack geometries, long wavelength approximation, and so on.

Numerical studies, which may be less restrictive, have also been introduced in order to investigate the effective elastic properties of cracked media. Saenger and Shapiro (2002) and Saenger et al. (2004) conducted finite difference (FD) simulations with rotated staggered grids (Saenger et al., 2000) in order to investigate the effective elastic properties of fractured 2D and 3D media, respectively. Dahm and Becker (1998) also calculated elastic moduli for media containing strongly interacting cracks using a boundary element method and a finite element method (FEM). Their results were in good agreement with the modified self- 
consistent theory predictions. However, models for numerical analysis need to be discretised with fine grids whose spatial scale is adjusted for representing small-scale cracks. In addition, the accommodation of model boundary or crack boundary shape remains as a problem in numerical methods such as finite difference schemes. The discretisation in space for cracked media remains a cause of inefficiency in simulations, for which some improvement has sought.

The estimation of the elastic properties of media can readily be accommodated in the simulation of seismic wave propagation. Seismic simulators using particle methods have been developed by many researchers (Toomey and Bean, 2000; Del Valle-Garcia and Sanchez-Sesma, 2003; O’Brien and Bean, 2004; Mariotti, 2007; O’Brien et al., 2009; Takekawa et al., 2012). Takekawa et al. (2012) applied a Hamiltonian particle method (HPM) (Suzuki and Koshizuka, 2008; Kondo et al., 2010) to simulate surface wave propagation. In HPM, the equation of motion of each particle is formulated in a Hamiltonian framework. The unique approach of HPM is that the deformation gradient tensor on each particle is decided by using the least-square method. A free surface is introduced in a simple way, by ending the interactions of particles at the surface. Since arbitrary refinement of spatial resolution is possible in a simple manner in the application of HPM (Takekawa et al., 2012), we are able to choose the optimum number of particles without unnecessarily consuming computational resources, and so enhance the efficiency of the simulation. If we use a local refinement technique in the FD method, we need special treatment around the refined area for the local fine grids (Aoi and Fujiwara, 1999). In FEM, we need a procedure to redefine the mesh for the refined area. Thus, the HPM should lead to a reduction of time taken for pre-processing. Furthermore, the accuracy of HPM for 
modelling of surface waves is higher than the finite difference method with rotated staggered grids (Takekawa et al., 2013). These features could allow us to efficiently calculate not only strong ground motion induced by earthquakes (Takekawa et al., 2012) but also the scattering of seismic waves by fractures (Okamoto et al., 2013) and other applications in material science and engineering (Saenger et al., 2012).

In this paper, we would like to propose HPM as a method to effectively estimate elastic properties of cracked media. We first explain our strategy for introducing the cracks in the rock mass and calculate the seismic wave field induced by a single crack in order to validate our strategy. We, then, investigate the validity of the particle method for determining the effective properties of cracked media comparing the numerical results with those from analytical approach. We then investigate the effect of the difference of spatial resolution around cracks on the effective velocity for efficient discretisation.

\section{METHOD}

\section{Hamiltonian particle method}

In HPM, a model for analysis is represented by an aggregate of particles. Each particle interacts with its nearby particles, and the extent of the interaction between particles is controlled by a weighting function $w(r)$. 
$w(r)=r_{\mathrm{e}} / r-1\left(r \leq r_{\mathrm{e}}\right), 0\left(r>r_{\mathrm{e}}\right)$

where $r$ is the distance between particles, and $r_{\mathrm{e}}$ is the radius of the influence domain. The influence domain between particles $i$ and $j$ is determined by the following equations.

$r_{\mathrm{e}}=\left(r_{\mathrm{e} i}+r_{\mathrm{e} j}\right) / 2$

$r_{\mathrm{e} i}=\alpha d_{i}$

where $r_{\mathrm{e} i}$ and $r_{\mathrm{e} j}$ are the influence domains of particles $i$ and $j, \alpha$ is a coefficient, and $d_{i}$ is the radius of the particle $i$. The calculation of the elastic wave propagation is based on the particle interactions between particles whose separations are less than $r_{\mathrm{e}}$.

The deformation gradient tensors at each particle position are defined to minimise the error function $e_{i}$ where,

$e_{i}=\sum_{j}\left|\mathbf{F}_{i} \mathbf{r}_{i j}^{0}-\mathbf{r}_{i j}\right|^{2} w_{i j}^{0}$

where $\mathbf{F}_{i}$ is the deformation gradient tensor at the position of particle $i, \mathbf{r}_{i j}^{0}$ and $\mathbf{r}_{i j}$ are the initial and current relative positions of particles $i$ and $j$, respectively, and $w_{i j}^{0}$ is the weighting value calculated by Eq. (1). The deformation gradient tensor $\mathbf{F}_{i}$ can be obtained by calculating the partial differential of Eq. (4) with respect to $\mathbf{F}_{i}$.

$\mathbf{F}_{i}=\sum_{j} \mathbf{r}_{i j} \otimes \mathbf{r}_{i j}^{0} w_{i j}^{0} \mathbf{A}_{i}^{-1}$ 
$\mathbf{A}_{i}=\sum_{j} \mathbf{r}_{i j}^{0} \otimes \mathbf{r}_{i j}^{0} w_{i j}^{0}$

We calculate strain and stress tensors as,

$\mathbf{E}=\left(\mathbf{F}^{\mathrm{T}} \mathbf{F}-\mathbf{I}\right) / 2$

$\mathbf{S}=2 \mu \mathbf{E}+\lambda \operatorname{tr}(\mathbf{E}) \mathbf{I}$

The total elastic strain and kinetic energy is as follows.

$$
\begin{aligned}
V & =\sum_{i}\left(\mathbf{E}_{i}: \mathbf{S}_{i} \Delta B_{i}\right) / 2 \\
K & =\sum_{i}\left(m_{i}\left|\mathbf{v}_{i}\right|^{2}\right) / 2
\end{aligned}
$$

where $\Delta B_{i}$ is the volume of particle $i, m_{i}$ is the mass of particle $i, \mathbf{v}_{i}$ is the velocity of particle $i$. Then we can calculate the equation of motion of the system:

$$
\begin{aligned}
& \rho_{i} \Delta B_{i} \partial \mathbf{v}_{i} / \partial \mathrm{t}=-\partial(V+K) / \partial \mathbf{x}_{i} \\
&=\sum_{j}\left(\mathbf{F}_{i} \mathbf{S}_{i} \mathbf{A}_{i}^{-1} \mathbf{r}_{i j}^{0} \Delta B_{i}+\mathbf{F}_{j} \mathbf{S}_{j} \mathbf{A}_{j}^{-1} \mathbf{r}_{i j}^{0} \Delta B_{j}\right) w_{i j}
\end{aligned}
$$

We apply a symplectic scheme, which is a time integration scheme conserving the total energy of Hamiltonian systems, as follows:

$$
\begin{aligned}
& \mathbf{v}\left(t+\frac{1}{2} \Delta t\right)=\mathbf{v}\left(t-\frac{1}{2} \Delta t\right)+\Delta t \cdot \partial \mathbf{v}(t) / \partial \mathrm{t} \\
& \mathbf{x}(t+\Delta t)=\mathbf{x}(t)+\Delta t \cdot \mathbf{v}\left(t+\frac{1}{2} \Delta t\right)
\end{aligned}
$$


Although the time updating process described above is very simple and the computational cost is comparable with the explicit Euler method, we can calculate particle motions with good energy conservation. On the other hand, it is known that high-frequency local oscillations occur, due to spurious singular modes that degrade numerical accuracy, although the total energy can be conserved. This results from the definition of the deformation gradient tensors only at the particles. Kondo et al. (2010) developed a suppression method by introducing an artificial potential force that has no physical meaning, with a coefficient to vary its effect. The coefficient of the artificial force (Eq. (14) in Kondo et al. (2010)) is decided by trial and error in this study. For numerical stability, we apply the Courant condition:

$\Delta t \leq \Delta x / V_{\max }$

where $\Delta t$ is the time step, $\Delta x$ is the minimum particle spacing and $V_{\max }$ is the maximum P-wave velocity in the model.

\section{Introduction of cracks}

In HPM, a free surface can be introduced just by setting the interactions between particles at the surface to zero (Suzuki et al., 2007; Takekawa et al., 2012). In this study, we take advantage of this feature to introduce traction-free boundaries inside the rock mass (i.e., cracks). Figure 1 shows the strategy of the introduction of a single crack, and the refinement of the spatial resolution only around the crack. The free surface is introduced just by cutting off the connectivity across the crack. This technique is simple to implement, but the reproducibility of the crack depends on the particle spacing. To ensure enough spatial resolution, we can replace a particle which is near the crack with four particles (Figure 
1 (b)). This procedure is also simple to implement. If we need finer particle spacing, we can further divide each quarter particle into four particles. In this way, arbitrary refinement of spatial resolution can be achieved in an effective manner. Figure 2 shows an example of particle refinement around cracks using the above approach. Since we use only distances between particles to discretise the model in HPM, it is possible to use different sizes of particles in an easy manner. Furthermore, because our model does not require high velocity contrasts to define cracks, this approach is stable like finite difference (FD) modelling (Saenger et al., 2000). Note that the time step should be decreased in accordance with the minimum particle spacing for stable calculations.

In order to validate the accuracy of our strategy for representing the shape of cracks, we conduct a numerical simulation of seismic wave scattering induced by a single crack. We use a result from a finite difference method with a rotated staggered grid (FDM-RSG) as a reference solution because the accuracy of FDM-RSG has already been validated by comparison with analytical solutions in Kruger et al. (2005). A numerical model is shown in Figure 3. A single crack, with inclination angle $30^{\circ}$ and length $1.61 \mathrm{~m}$, is embedded in the centre of the model. Since the particle and grid spacing in HPM and FDM-RSG are 2 $\mathrm{mm}$, the crack is represented by more than 800 particles or grid nodes. The upper and lower boundaries of the model are periodic boundaries. A plane P-wave is generated at the right edge of the model, and propagates through the model. We record the scattering waves induced by the crack using a receiver array placed at the right side of the crack. Figure 4 shows a snapshot of the disturbed wave field calculated by HPM. We can observe that reflected $\mathrm{P}$ - and $\mathrm{S}$-waves are generated after the incident plane $\mathrm{P}$-wave hit the crack. Figure 5 shows the waveforms observed at the receiver array. Each waveform agrees well with the reference traces, for both vertical and horizontal components. This indicates that our 
strategy can calculate scattering waves induced by the crack accurately if we use a large number of particles to represent the crack. The number of particles required to represent the crack with sufficient accuracy is discussed in the next section.

\section{NUMERICAL RESULTS OF EFFECTIVE WAVE VELOCITIES}

\section{Model set-up}

We use numerical models as shown in Figure 6. The model has a cracked region in the centre of it. The cracked region is $0.2 \mathrm{~m} \times 0.2 \mathrm{~m}$, and has randomly placed, randomly oriented, rectilinear, dry and non-intersecting cracks. All cracks have the same length, i.e., $2 l_{k}=0.0061 \mathrm{~m}$, where $l_{k}$ represents the half-length of each crack. The cracked region is set into a 2D isotropic elastic medium with a P-wave velocity of $V_{p}=4000 \mathrm{~m} / \mathrm{s}$, an $\mathrm{S}$-wave velocity of $V_{s}=2310 \mathrm{~m} / \mathrm{s}$, and a mass density of $\rho=2700 \mathrm{~kg} / \mathrm{m}^{3}$. A plane wave $(\mathrm{P}$ - or $\mathrm{SV}$-wave) is incident from the right side of the model and goes through the cracked region. We detect the plane wave passing through the cracked region with a line of receivers placed just to the left of the cracked region. The source wavelet in our numerical experiments is the first derivative of a Gaussian function, with a dominant frequency of $20 \mathrm{kHz}$. Therefore, the dominant wavelengths of P- and SV-wave in the homogeneous region are $0.2 \mathrm{~m}$ and $0.1155 \mathrm{~m}$, respectively. This means that our numerical conditions are a good approximation to the long wavelength limit. The background particle spacing is $0.001 \mathrm{~m}$ and the time step is $0.01 \mu \mathrm{s}$. We apply periodic boundary conditions in the vertical direction. 
We describe the number of cracks in the cracked region with the crack density parameter $\phi$, defined by:

$\phi=\frac{1}{A} \sum_{k=1}^{n} l_{k}^{2}$

where $A$ represents the area of the cracked region, and $n$ the number of cracks. Figure 7 shows typical examples of cracked regions with different crack densities.

Figure 8 shows examples of the spatial-averaged waveforms recorded at the receiver array for different crack densities. We used 200 receivers to calculate the averaged waveforms. It can be observed that the time delay of the peak of the spatial-averaged waveforms increases with increasing crack density. Using this time delay, we can estimate the effective velocity in the cracked region.

\section{Effective P- and SV-wave velocities}

Figure 9 shows our numerical results of effective velocities. For comparison, the predictions of the effective velocities from three of the theories described in the Introduction are also shown. Six numerical experiments were conducted at each crack density with different crack distributions. Note that it is difficult to detect error bars in Figure 9 (b) 
because the standard deviations are very small. We refined the spatial resolution around cracks in two steps, as in Figure 2. The effect of the level of refinement around cracks on the effective velocity will be investigated in the next section. In Figure 9, we observe the relative decrease in the normalised velocities as a function of the crack density. For a sparse crack distribution (e.g. $\phi=0.01$ ), the numerical and three analytical results are in good agreement with each other. This is one of the arguments for the validity of our numerical method. For a dense crack distribution, on the other hand, our numerical results for P- and SV-wave velocities are in good agreement with the predictions of the modified self-consistent theory. We will discuss the validity of our results in response to the results of the next section with comparing to the previous studies.

\section{Effect of the ratio of the particle spacing to the crack length}

In this section, we examine the effect of the number of particles per crack length. Fine spacing of particles improves the accuracy of the calculation, but increases computational costs, i.e., memory and CPU time. For both reasonable accuracy and computational costs, it is important to ascertain the appropriate level of refinement around cracks. The ratio of the crack length to the particle spacing is defined by a parameter $\xi$,

$\xi=\frac{2 l_{k}}{\Delta x}$ 
where $\Delta x$ is the particle spacing around cracks. Incidentally, in the previous section, the crack length and particle spacing around cracks are $0.0061 \mathrm{~m}$ and $0.00025 \mathrm{~m}$, respectively, so that the parameter $\xi$ was 24.4 .

We also define the "nominal" crack length $K_{\mathrm{N}}$ and the "real" crack length $K_{\mathrm{R}}$ as shown in Figure 10. Since a crack is introduced by cutting the connectivity between particles that intersect the crack, the reproducibility of the crack length depends on the spatial resolution around cracks. If we use a coarse arrangement of particles as in Figure 10 (a), the difference between $K_{\mathrm{N}}$ and $K_{\mathrm{R}}$ will be larger than with the use of a finer arrangement (Figure 10 (b)). Therefore, refining the spatial resolution provides a more accurate representation of the crack length. Furthermore, a fine particle arrangement can reproduce significantly thin cracks, as are assumed in the theories, more accurately than a coarse distribution. To test these effects on the effective velocity, we use a special arrangement of cracks as shown in Figure 11. Each crack length is set to $0.002999 \mathrm{~m}$ and the regular background particle spacing is set to $0.001 \mathrm{~m}$. All cracks are aligned with the regular particle lattice. In this case, both the crack length measures, $K_{\mathrm{N}}$ and $K_{\mathrm{R}}$, are approximately same. The crack density $\phi$ is fixed at 0.05 and the elastic parameters are also the same as those in the previous section. We change the parameter $\xi$ from 3 to 48 by changing the particle spacing around cracks, and calculate the effective P-wave velocities of the cracked media.

The calculation results for different $\xi$ are shown in Figure 12 as filled circles. Each symbol is an average value over six model realisations. The effective P-wave velocities are very slow comparing with the results of Figure 9 because all of the cracks are perpendicular 
to the direction of the incident wave. The calculated velocity decreases with an increase in $\xi$. Figure 13 shows the horizontal normal stress field around the cracks for two different values of $\xi$. The incident wave propagates from right to left. In both figures, the stress concentration can be observed around the tips of the vertical cracks, but each case shows a different degree of stress concentration. Since the coarse arrangement of particles cannot reproduce the stress field at the crack tips as accurately as the fine arrangements, the stress fields just around the crack tips are quite different from each other. This induces an artificial stiffness that results in larger effective velocities, as indicated by previous studies (e.g. Dahm and Becker, 1998). As we increase the number of particles around the cracks, the results converge to a certain value.

Next, we change the crack length from $0.002999 \mathrm{~m}$ to $0.003001 \mathrm{~m}$. In this case, the "real" crack length $K_{\mathrm{R}}$ is much greater than the "nominal" crack length $K_{\mathrm{N}}$ when we use the coarse particle arrangement. The distribution of cracks is the same as Figure 11. The calculated effective P-wave velocities are shown in Figure 12 as open squares. The effective velocity increases with an increase in $\xi$. When we set the crack length to $0.003001 \mathrm{~m}$, not only the effect of the difference between $K_{\mathrm{N}}$ and $K_{\mathrm{R}}$ but also the effect of the artificial stiffness is included in the model. Nevertheless, the effective velocity is underestimated when we use coarse particles. This means that the underestimation due to the difference between $K_{\mathrm{N}}$ and $K_{\mathrm{R}}$ has a larger effect than the overestimation due to the artificial stiffness. We note that the difference between $K_{\mathrm{N}}$ and $K_{\mathrm{R}}$ in this model has a major influence on the underestimation because the cracks are aligned with the regular latticed particle arrangement. Using fine particles reduces the difference between the results for this model and those for the crack 
length of $0.002999 \mathrm{~m}$. The difference in the normalised effective velocity between the two cases is approximately $1.1 \%$ at $\xi$ of 24 .

In summary, a coarse particle arrangement acts to both increase and decrease the effective velocity. In an arbitrary arrangement of cracks, both randomly distributed and randomly oriented, the effective velocity could be strongly affected by both effects.

In order to demonstrate these effects in arbitrary arrangements, we use the same model as shown in Figure 6. The crack length is fixed at $0.0061 \mathrm{~m}$. The particle spacing in the noncracked region is $0.002 \mathrm{~m}$. The crack density $\phi$ is fixed to 0.05 . We make six different crack distributions for each parameter $\xi$ and averaged the results. The numerical result is shown in Figure 14. It can be observed that the effective velocity slightly increases with increasing parameter $\xi$. The effective velocity is higher than shown in Figure 12 in spite of the same crack density in the model. This is because the cracks are not all aligned perpendicular to the direction of the incident wave. The variation in the effective velocities with respect to $\xi$ in Figure 14 is smaller than those in Figure 12. Since the coarse particle arrangement may act to increase or decrease the effective velocity, as described above, the contributions could cancel each other out. These results indicate that we can obtain a stable prediction of the effective velocities using the average of values from several model realisations. However, the standard deviation in case of low $\xi$ remains high. In Figure 12, the difference in the normalised effective velocity between the two cases decreased to about 1 per cent at a $\xi$ of 24 . This indicates that a fine particle arrangement, with more than 
approximately 20 particles per crack length, will provide a stable prediction of effective velocities even with extreme crack distributions like that shown in Figure 11.

\section{Comparison to the previous studies}

In the previous section, we compared our numerical results with several theoretical ones; the self-consistent theory, the theory for non-interacting cracks and the modified self-consistent theory. Our results of $\mathrm{P}$ - and $\mathrm{SV}$-wave velocities are in good agreement with the predictions of the modified self-consistent theory (Figure 9). In the self-consistent theory, the bulk and shear moduli become negative and Poisson's ratio exceeds 0.5 at high crack densities. In the theory for non-interacting cracks, crack-to-crack interactions are neglected. In contrast, the modified self-consistent theory provides reasonable solutions for effective properties lying in the physical range at high crack densities, and does consider crack-to-crack interactions.

In previous numerical studies, numerical solutions for the effective velocities using the modified self-consistent theory using FD simulations (Saenger and Shapiro, 2002; Saenger et al., 2004) agreed well with solutions using boundary element and finite-element simulations (Dahm and Becker, 1998). Our results are in good agreement with their conclusions. On the other hand, Davis and Knopoff (1995), Grechka and Kachanov (2006) and Grechka (2007a) proposed that the interactions between cracks can be neglected at high crack densities, and that the theory for non-interacting cracks performs well. Furthermore, Grechka (2007b) pointed out ambiguity in the consistency and reproducibility of FD 
simulations (e.g., Saenger et al., 2004), which stems from the difference between the "nominal" and "real" crack lengths. However, the effective velocity in Figure 12 and 14 converged to a constant value as $\xi$ increased. This indicates that the definition of the crack length does not have large influence on the effective velocity if we use enough particles per crack length. Thus, we conclude that our results eliminated the ambiguity over the definition of the crack length, and support the correctness of the modified self-consistent theory.

\section{CONCLUSIONS}

We have presented an application of HPM for calculating seismic wave propagation and evaluating effective velocities in cracked media. HPM has some advantages in representing cracks inside the elastic media: i) it is easy to introduce free surfaces, ii) arbitrary spatial refinement is possible in a simple and easy manner.

In order to investigate the effectiveness of the method, we considered 2D isotropic cracked media. We predicted the effective P- and SV-wave velocity and compared them with values predicted by three different theoretical approaches. Our results were in good agreement with the effective velocities from the modified self-consistent theory. This was consistent with the previous numerical studies conducted by the finite difference method, the finite element method and the boundary element method. We also investigated the influence of the ratio of crack length to particle spacing on the effective velocity. The result indicated that we could obtain stable results with values of more than 20 for the parameter $\xi$. 
In this study, we placed limitations on the model styles, so that cracks did not intersect each other, and crack length is much shorter than the wavelength. These restrictions were applied to enable comparison with theoretical results obtained under similar conditions. In numerical studies, however, there is no necessary restriction on crack intersections or wavelengths. Therefore, numerical experiments using HPM could also support estimation of the effective velocities under various conditions that are not available to most theoretical approaches.

\section{ACKNOWLEDGEMENTS}

This work was partly supported by a research grant from Arai Science and Technology Foundation. We thank the editor and two anonymous reviewers for their careful revision that enabled improving the manuscript significantly.

\section{REFERENCES}

Aoi, S., Fujiwara, H., 1999, 3D finite-difference method using discontinuous grids, Bulletin of the Seismological Society of America, 89, 918-930.

Budiansky, B. and O’Connell, R., 1976, Elastic moduli of a cracked solid, International Journal of Solids and Structures, 12, 81-97.

Dahm, T. and Becker, TH., 1998, On the elastic and viscous properties of media containing strongly interacting in-plane cracks: Pure and Applied Geophysics, 151, 1-16. 
Davis, P. M. and Knopoff, L., 1995, The elastic modulus of media containing strongly interacting antiplane cracks, Journal of Geophysical Research, 100, 18253-18258.

Del Valle-Garcia, R., Sanchez-Sesma, F. J., 2003, Rayleigh waves modeling using an elastic lattice model, Geophysical Research Letters, 30, 1866.

Grechaka, V. and Kachanov, M., 2006, Effective elasticity of rocks with closely spaced and intersecting cracks, Geophysics, 71, D85-D91. DOI: 10.1190/1.2197489

Grechka, V., 2007a, Comparison of the non-interaction and differential schemes in predicting the effective elastic properties of fractured media, International Journal of Fracture, 144, 181-188. DOI: 10.1007/s10704-007-9088-Z

Grechka, V., 2007b, Reply to comment by E. H. Saenger, International Journal of Fracture, 146, 293-294. DOI: 10.1007/s10704-007-9136-8

Henyey, F. S. and Pomphrey, N., 1982, Self-consistent elastic moduli of a cracked solid, Geophysical Research Letters, 9, 903-906.

Kondo, M., Suzuki, Y., Koshizuka, S., 2010, Suppressing local particle oscillations in the Hamiltonian particle method for elasticity, International Journal for Numerical Methods in Engineering, 81, 1514-1528. DOI: 10.1002/nme.2744

Kuruger, O. S., Saenger, E. H., Shapiro, S. A., 2005, Scattering and diffraction by a single crack: an accuracy analysis of the rotated staggered grid, Geophysical Journal International, 162, 25-31. DOI: 10.1111/j.1365-246X.2005.02647.x

Mariotti, C., 2007, Lamb's problem with the lattice model Mka3D, Geophysical Journal International, 171, 857-864. DOI: 10.1111/j.1365-246X.2007.03579.x 
O’Brien, G. S., Bean, C. J., 2004, A 3D discrete numerical elastic lattice method for seismic wave propagation in heterogeneous media with topography, Geophysical Research Letters, 31, L14608. DOI: 10.1029/2004GL020069

O’Brien, G. S., Bean, C. J., Tapamo, H., 2009, Dispersion analysis and computational efficiency of elastic lattice methods for seismic wave propagation, Computers and Geosciences, 35, 1768-1775. DOI: 10.1016/j.cageo.2008.12.004

O’Connell, R. and Budiansky, B., 1974, Seismic velocities in dry and saturated cracked solids, Journal of Geophysical Research, 79, 5412-5426.

Okamoto, K., Mikada, H., Goto, T. and Takekawa, J., 2013, Numerical analysis of the relationship between time-variant coda-Q and the variation in crustal stress, Geophysical Journal International, 195, 575-581. doi: 10.1093/gji/ggt243.

Saenger, E. H., Gold, N. and Shapiro, S. A., 2000, Modeling the propagation of elastic waves using a modified finite-difference grid, Wave Motion, 31, 77-92.

Saenger, E. H. and Shapiro, S. A., 2002, Effective velocities in fractured media: a numerical study using the rotated staggered finite-difference grid, Geophysical Prospecting, 50, 183194.

Saenger, E. H., Kruger O. S. and Shapiro, S. A., 2004, Effective elastic properties of randomly fractured soils: 3D numerical experiments: Geophysical Prospecting, 52, 183-195. DOI: $10.1111 / \mathrm{j} .1365-2478.2004 .00407 . x$

Saenger, E. H., Uribe, D., Janicke, R., Ruiz, O. and Steeb, H., 2012, Digital material laboratory: Wave propagation effects in open-cell aluminium foams, International Journal of Engineering Science, 58, 115-123. DOI: 10.1016/j.ijengsci.2012.03.030 
Suzuki, Y., Koshizuka, S., Oka, Y., 2007, Hamiltonian moving-particle semi-implicit (HMPS) method for incompressible fluid flows, Computer Methods in Applied Mechanics and Engineering, 196, 2876-2894. DOI: 10.1016/j.cma.2006.12.006

Suzuki, Y., Koshizuka, S., 2008, A Hamiltonian particle method for non-linear elastodynamics, International Journal for Numerical Methods in Engineering, 74, 1344-1373. DOI: $10.1002 / \mathrm{nme} .2222$

Takekawa, J., Madariaga, R., Mikada, H. and Goto, T., 2012, Numerical simulation of seismic wave propagation produced by earthquake by using a particle method, Geophysical Journal International, 191, 1305-1316. DOI: 10.1111/j.1365-246X.2012.05676.x

Takekawa, J., Mikada, H. and Goto, T., 2013, Accuracy of a particle method for modelling of Rayleigh waves, Butsuri-Tansa, 66, 85-95. (in Japanese with English abstract)

Toomey, A., Bean, C. J., 2000, Numerical simulation of seismic waves using a discrete particle scheme, Geophysical Journal International, 141, 595-604. 


\section{FIGURE CAPTIONS}

\section{Fig. 1:}

The schematic figure of the introduction of a crack. Solid circles are particles, the dotted line is a crack, and the solid lines represent connectivity between particles. A free surface inside the elastic medium is introduced by cutting off the connectivity that intersects the surface. (a) Original particle arrangement, (b) particle arrangement after refinement of spatial resolution in the vicinity of the crack.

\section{Fig. 2:}

An example of particle arrangement after the refinement of spatial resolution in the vicinity of cracks. Circles and solid lines represent particles and cracks, respectively. Only particles around cracks are replaced by smaller particles. In this figure, a two step refinement is depicted.

\section{Fig. 3:}

Model set-up for numerical validation. White circles represent receivers and the solid line represents the crack.

\section{Fig. 4:}

A snapshot of the horizontal displacement velocity calculated by HPM. 


\section{Fig. 5:}

The waveforms recorded by the receiver array. Solid and dashed lines are the waveforms from HPM and FDM-RSG calculations, respectively. The dotted line represents the difference between the waveforms but the scale is amplified by a factor of 10. (a) horizontal and (b) vertical displacement velocities.

\section{Fig. 6:}

Numerical model set-up for the calculation of effective velocities. White triangles represent receivers.

\section{Fig. 7:}

Examples of crack distributions. The solid square and solid lines represent the cracked region and cracks, respectively. (a) $\phi=0.05$, (b) $\phi=0.10$, (c) $\phi=0.20$.

\section{Fig. 8:}

Displacement waveforms calculated at the receivers for different crack densities.

\section{Fig. 9:}


Normalised effective velocities versus crack density. $\quad V_{\text {eff }}$ and $V$ represent the effective velocity and background velocity, respectively. Circles are numerical results, averaged over six repetitions with different crack distributions. Solid lines represent the result of the theory for non-interacting cracks, dotted lines represent that of self-consistent theory, and dot-dash lines represent that of the modified self-consistent theory.

(a) P-wave velocity, (b) SV-wave velocity.

\section{Fig. 10:}

The definition of "nominal" crack length $K_{\mathrm{N}}$ and the "real" crack length $K_{\mathrm{R}}$.

\section{Fig. 11:}

Crack distribution and crack alignment used for the demonstration of the effect of the resolution around cracks.

\section{Fig. 12:}

Normalised effective velocities calculated for the special arrangement as shown in Figure 11.

\section{Fig. 13:}

Snapshots of the horizontally normal stress field. (a) $\xi=3$, (b) $\xi=24$. 


\section{Fig. 14:}

Normalised effective P-wave velocity versus parameter $\xi$ calculated for the random arrangement of cracks as shown in Figure 6. The error bars denote the standard deviation for different crack distributions. 


\section{EQUATIONS}

$$
\begin{aligned}
& w(r)=r_{\mathrm{e}} / r-1\left(r \leq r_{\mathrm{e}}\right), 0\left(r>r_{\mathrm{e}}\right) \\
& r_{\mathrm{e}}=\left(r_{\mathrm{e} i}+r_{\mathrm{e} j}\right) / 2 \\
& r_{\mathrm{e} i}=\alpha d_{i} \\
& e_{i}=\sum_{j}\left|\mathbf{F}_{i} \mathbf{r}_{i j}^{0}-\mathbf{r}_{i j}\right|^{2} w_{i j}^{0} \\
& \mathbf{F}_{i}=\sum_{j} \mathbf{r}_{i j} \otimes \mathbf{r}_{i j}^{0} w_{i j}^{0} \mathbf{A}_{i}^{-1} \\
& \mathbf{A}_{i}=\sum_{j} \mathbf{r}_{i j}^{0} \otimes \mathbf{r}_{i j}^{0} w_{i j}^{0} \\
& \mathbf{E}=\left(\mathbf{F}^{\mathrm{T}} \mathbf{F}-\mathbf{I}\right) / 2 \\
& \mathbf{S}=2 \mu \mathbf{E}+\lambda \operatorname{tr}(\mathbf{E}) \mathbf{I} \\
& V=\sum_{\mathrm{i}}\left(\mathbf{E}_{\mathrm{i}}: \mathbf{S}_{\mathrm{i}} \Delta \mathrm{B}_{\mathrm{i}}\right) / 2 \\
& \mathrm{~K}=\sum_{i}\left(m_{i}\left|\mathbf{v}_{i}\right|^{2}\right) / 2 \\
& \rho_{i} \Delta B_{i} \partial \mathbf{v}_{i} / \partial t=-\partial(V+K) / \partial \mathbf{x}_{i} \\
& =\sum_{j}\left(\mathbf{F}_{i} \mathbf{S}_{i} \mathbf{A}_{i}^{-1} \mathbf{r}_{i j}^{0} \Delta B_{i}+\mathbf{F}_{j} \mathbf{S}_{j} \mathbf{A}_{j}^{-1} \mathbf{r}_{i j}^{0} \Delta B_{j}\right) w_{i j} \\
& \mathbf{v}(t+\Delta t)=\mathbf{v}(t)+\Delta t \cdot \partial \mathbf{v}(t+\Delta t) / \partial t \\
& \mathbf{x}(t+\Delta t)=\mathbf{x}(t)+\Delta t \cdot \mathbf{v}(t) \\
& \Delta t \leq \Delta x / V_{\max } \\
& \phi=\frac{1}{A} \sum_{k=1}^{n} l_{k}^{2} \\
& \xi=\frac{2 l_{k}}{\Delta x}
\end{aligned}
$$




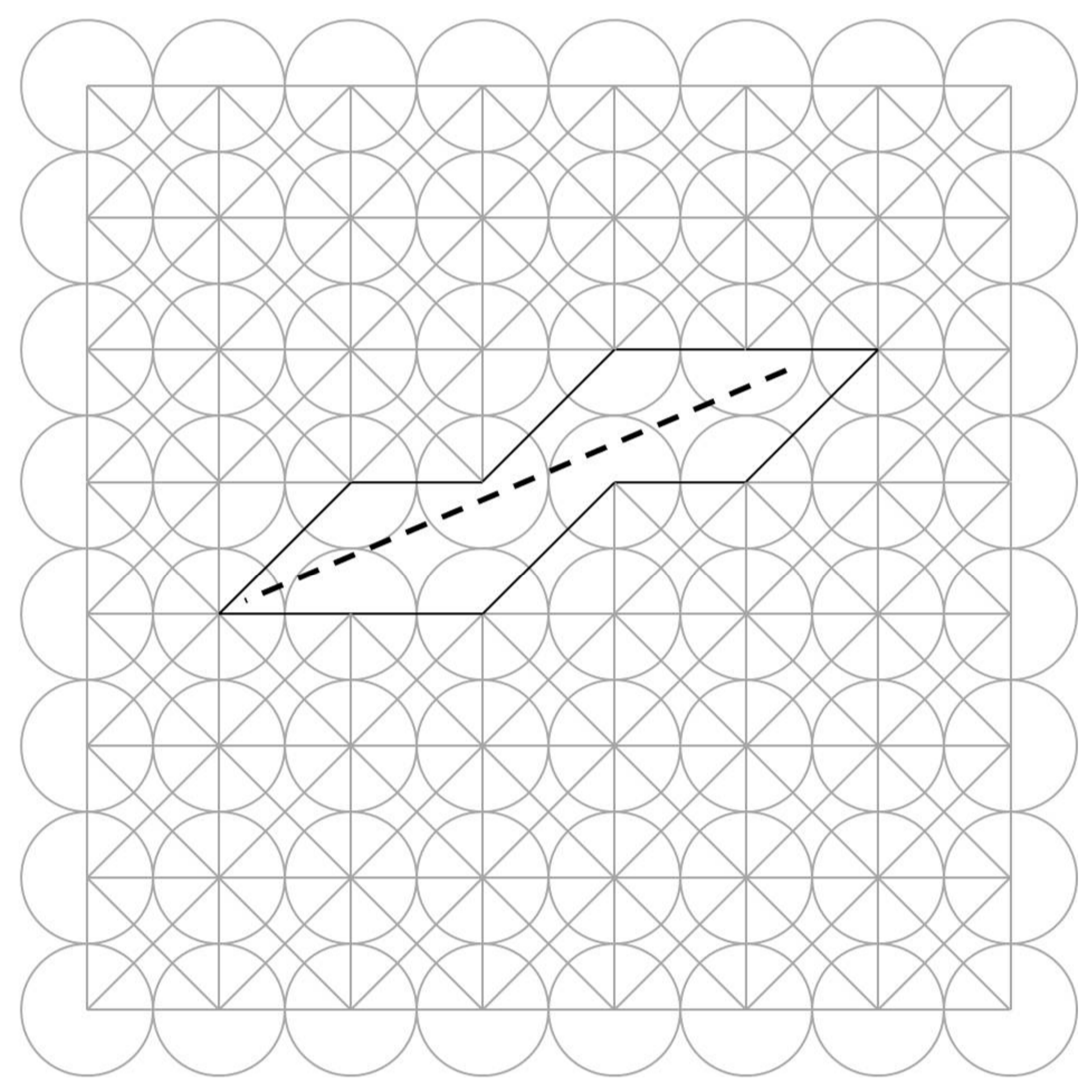

Fig. 1(a) 


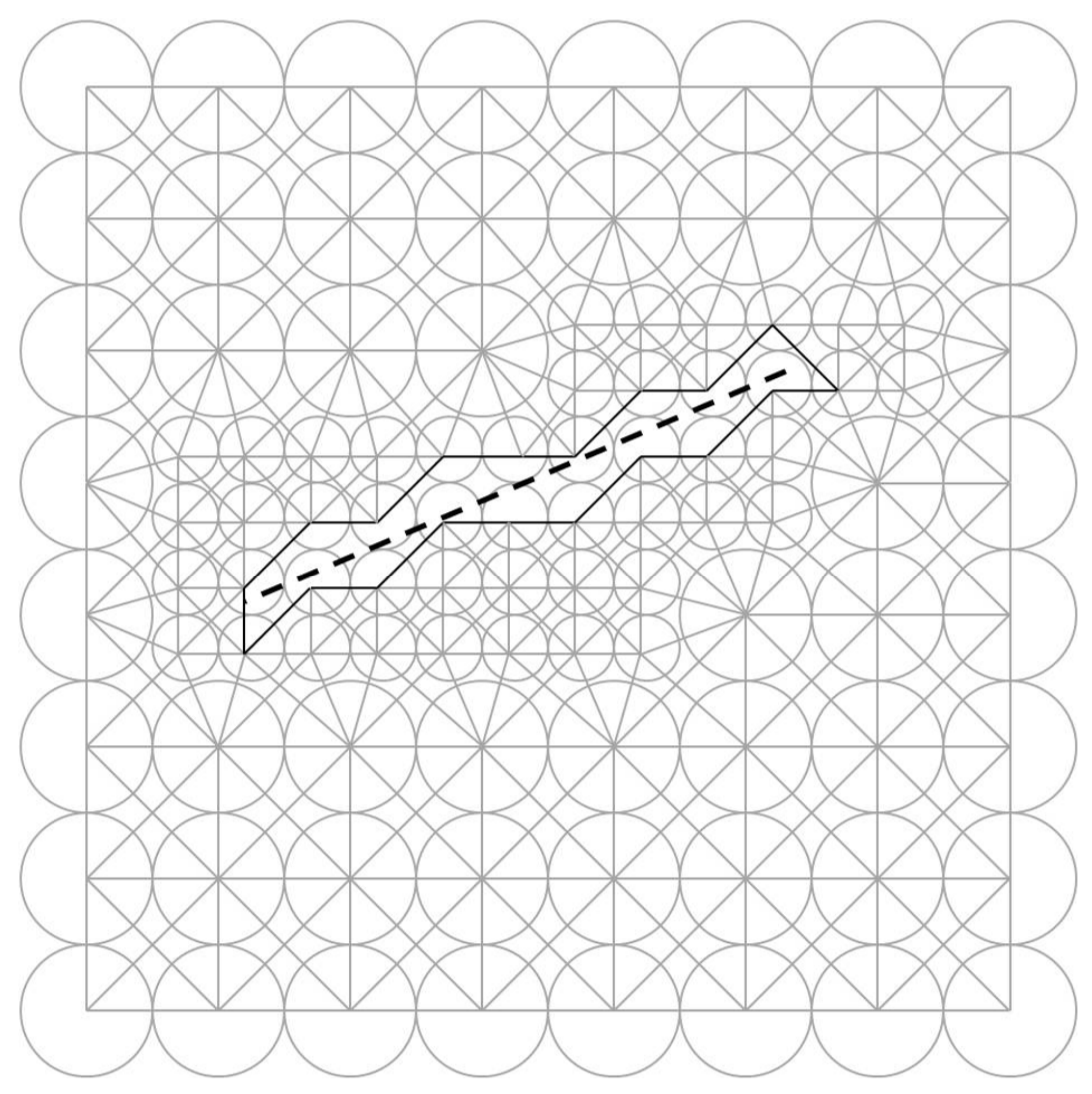

Fig. 1(b) 
$+\infty+1+1+1$

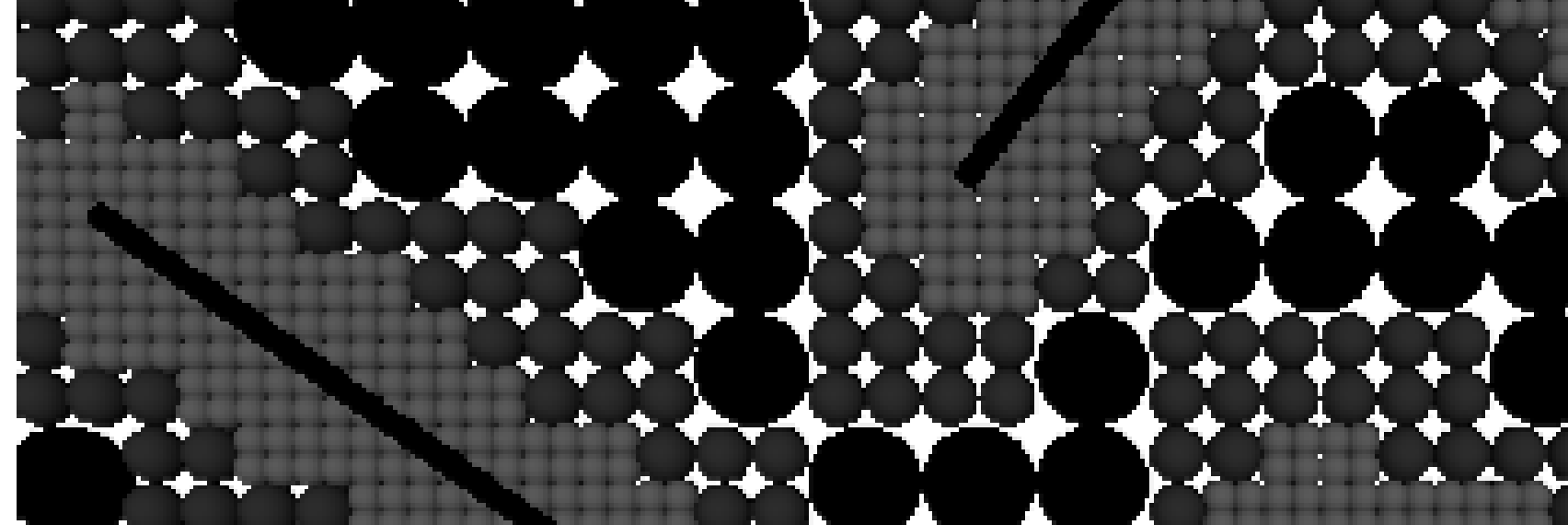

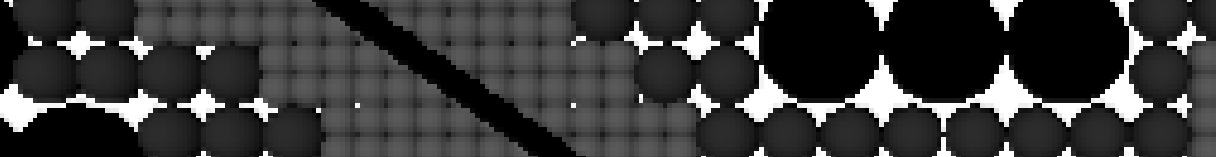

$$
\begin{aligned}
& +4+\ldots+1,+4+4+1
\end{aligned}
$$

$+4$

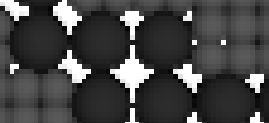

$-4-4+4$

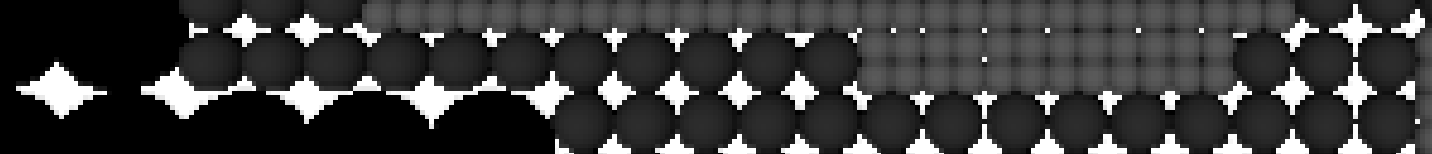

$1+4+4+40+4+404$

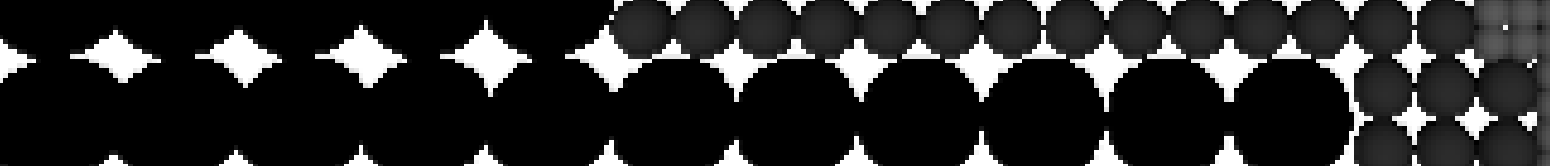

$(t+t+t+t+t+1+t$

$+2+t+4+t+4+1$

$+4+t+t+t+t+1$

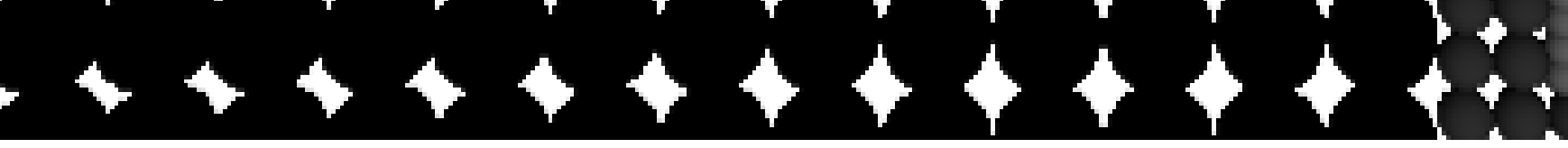

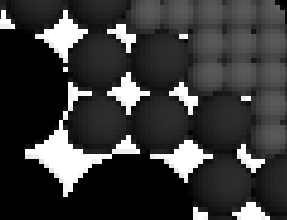

$4+4$

$\therefore+\cdots+1+1+1+$

\section{$+1$} $+$ $+4+4$ $1+4: 1:+4+$ $+4+1+1+1+1+1+1+1+1+1+1+4$ $+4+4+4+4-4$ $+4+4+1$ $+4$ $\therefore+4+\infty+1+$

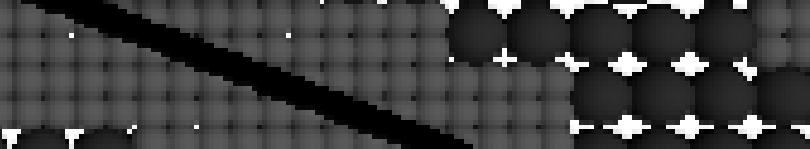
$+4+++1: 11: 1:+t+-$ $+4+++++1+1+4$ $+4+\infty+1+\infty+1$ $+4+4+4+4$ $+4$ $1+4+4+4$ $+1+4+4$
$1+1+1+4$
$1+1+4$ Fig. 2 


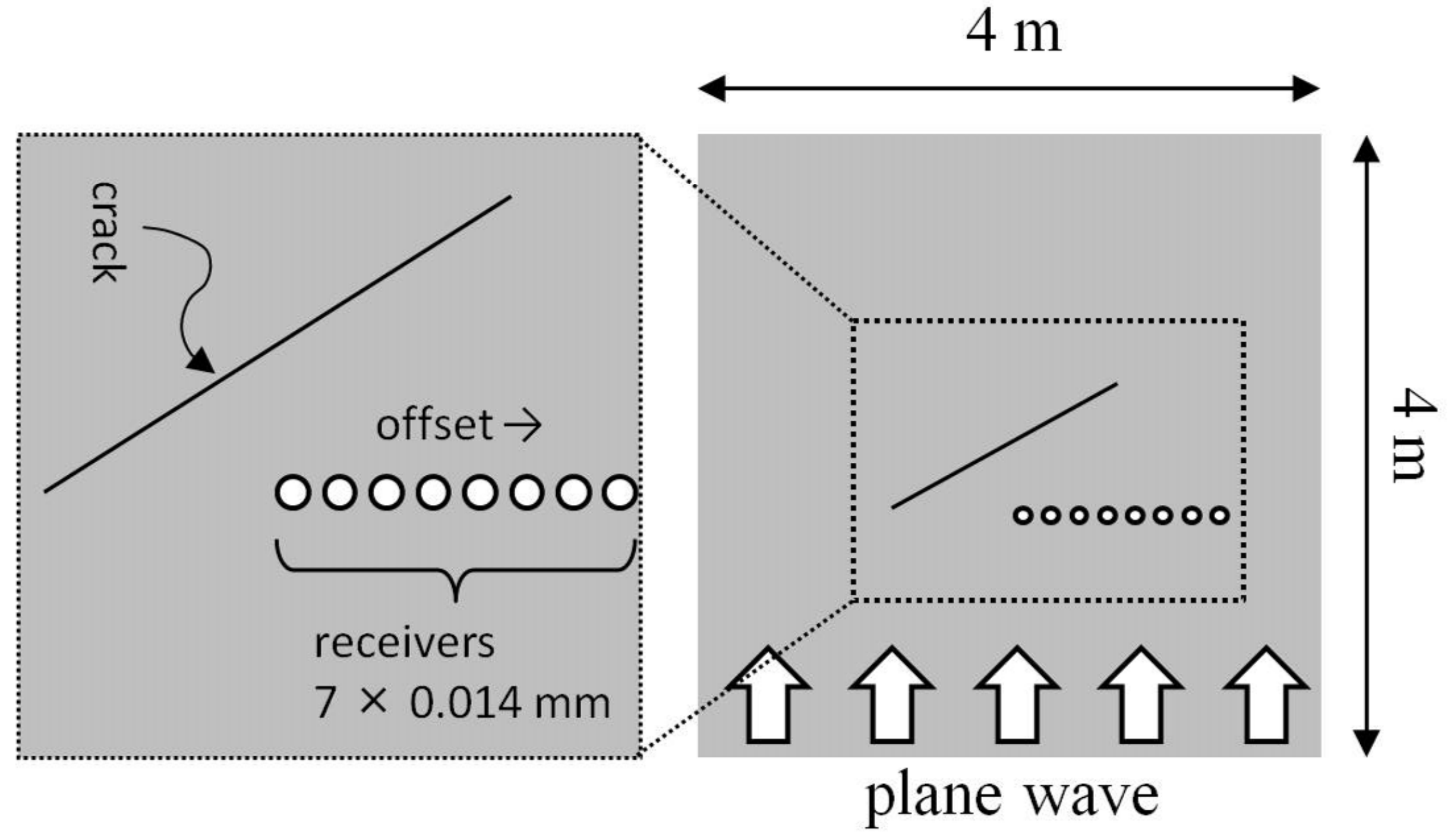

Fig. 3 

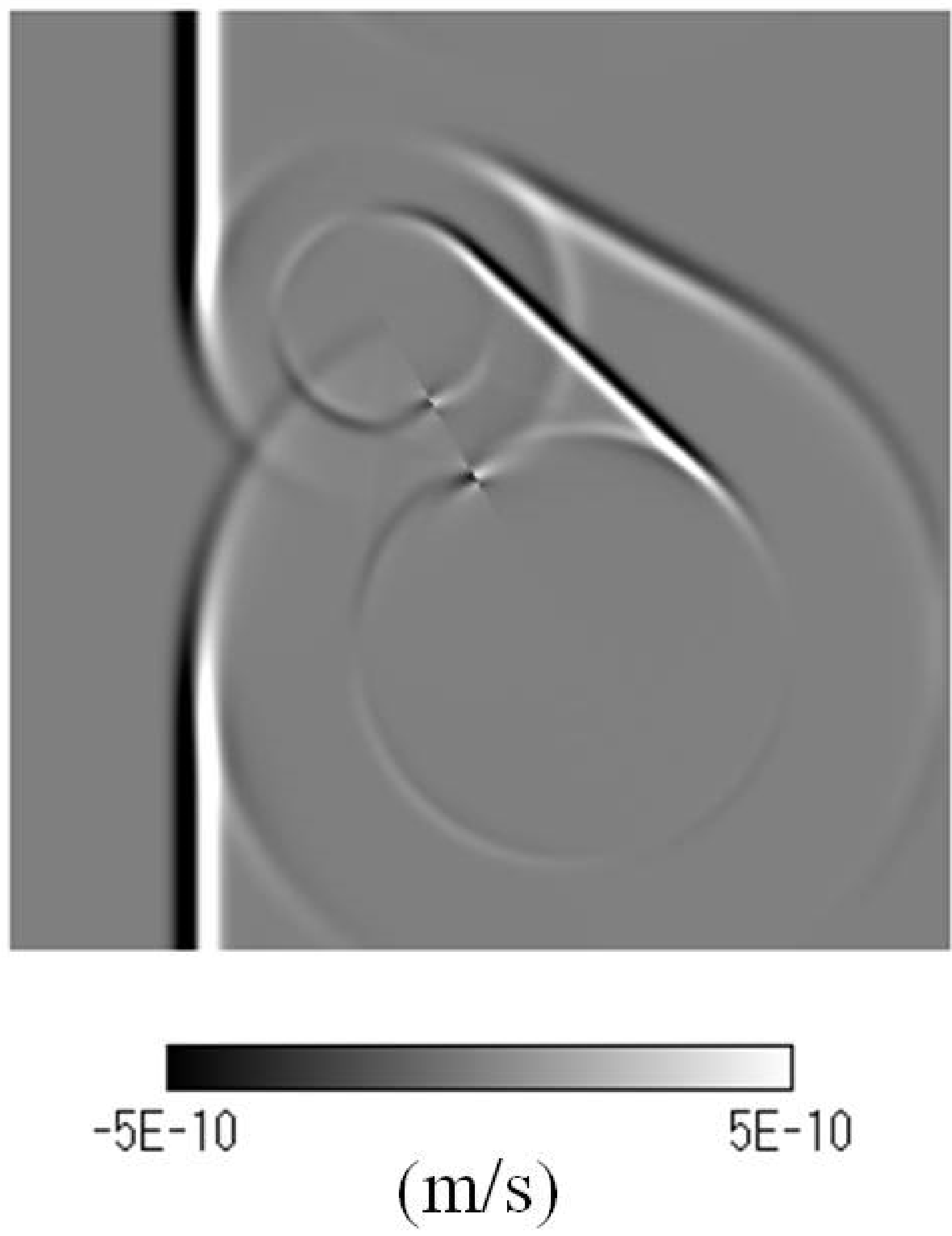

Fig. 4 


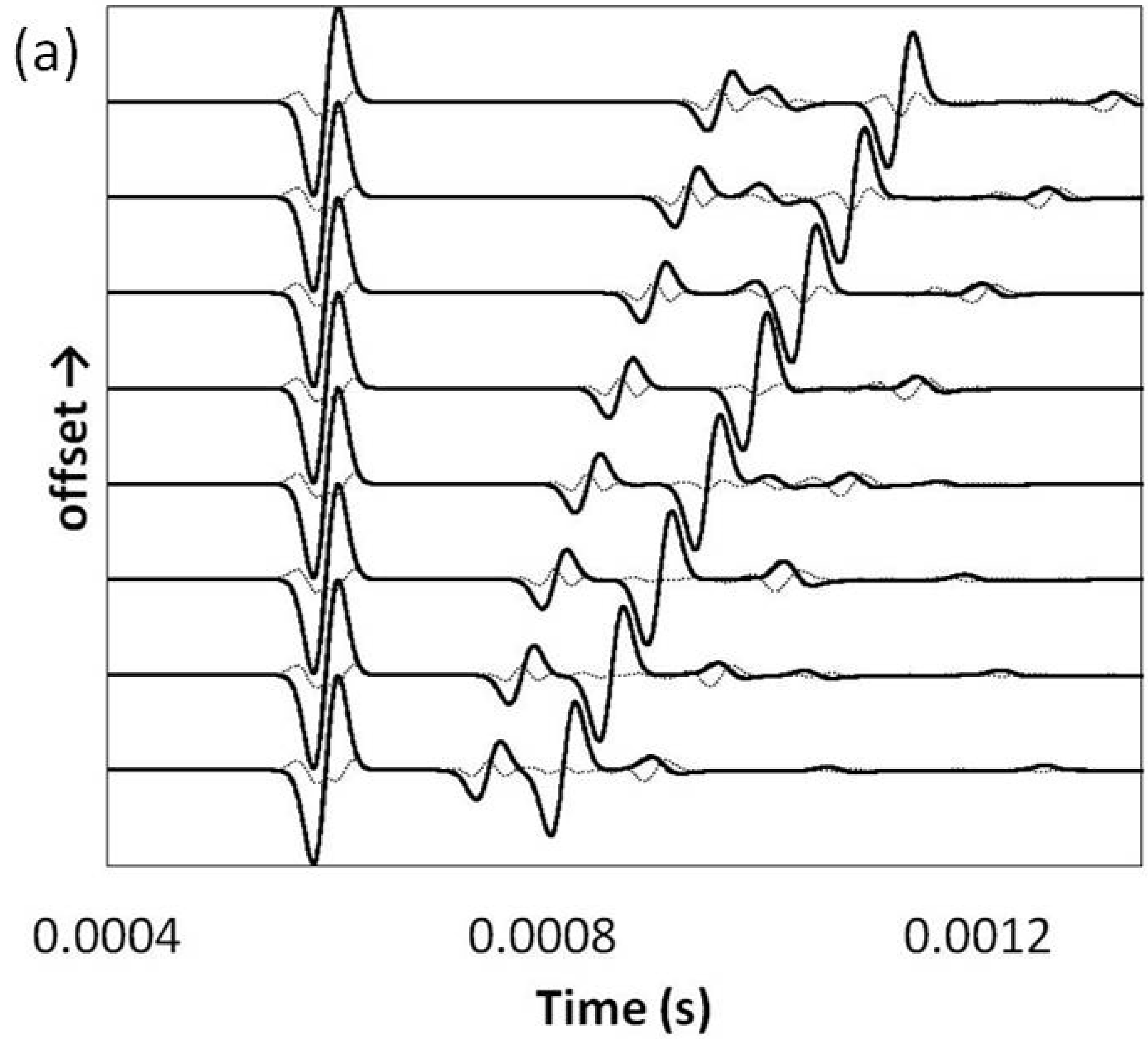

Fig. 5(a) 


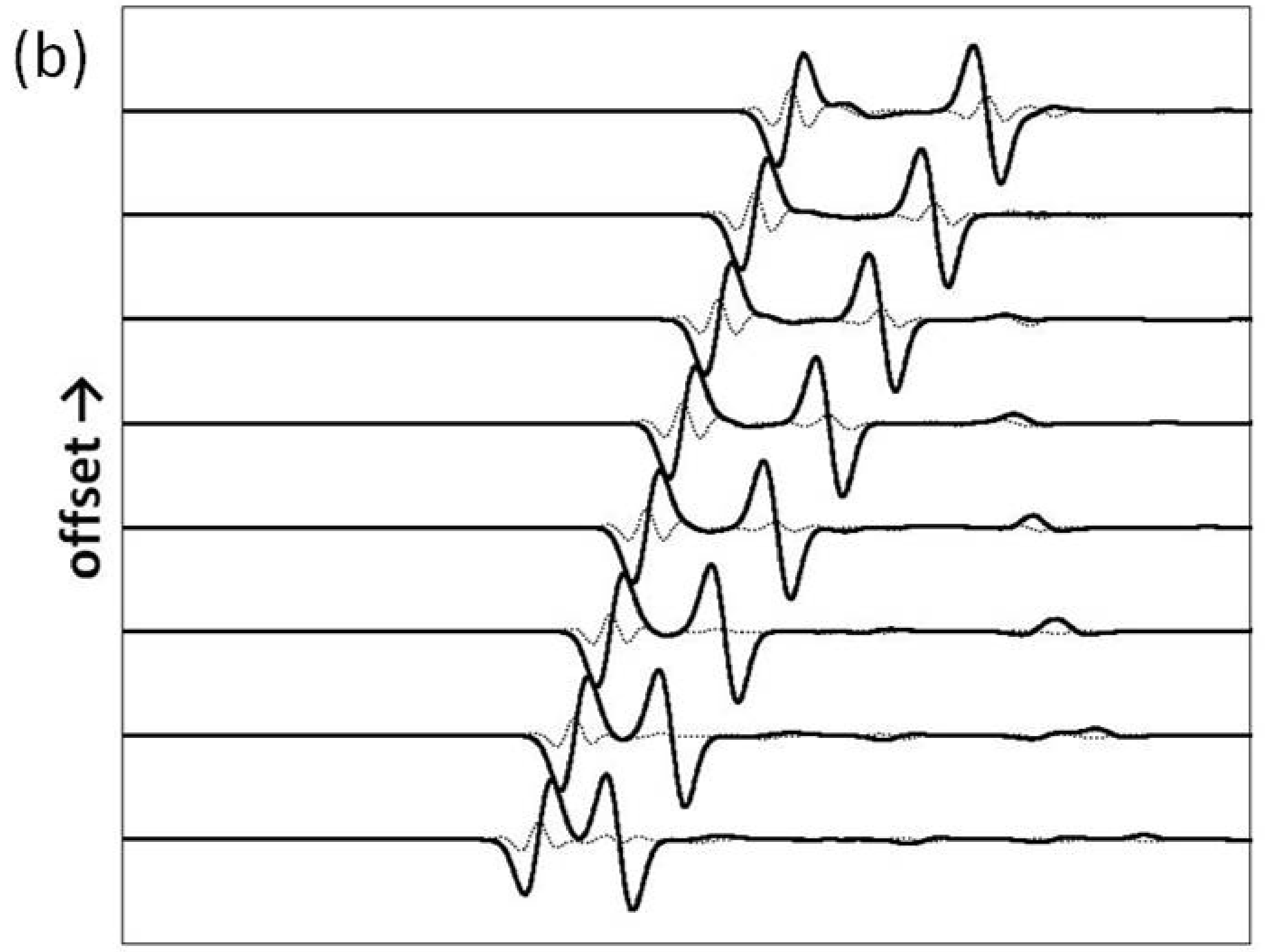

0.0004

0.0008
Time (s)

0.0012

Fig. 5(b) 


\section{cracked region}
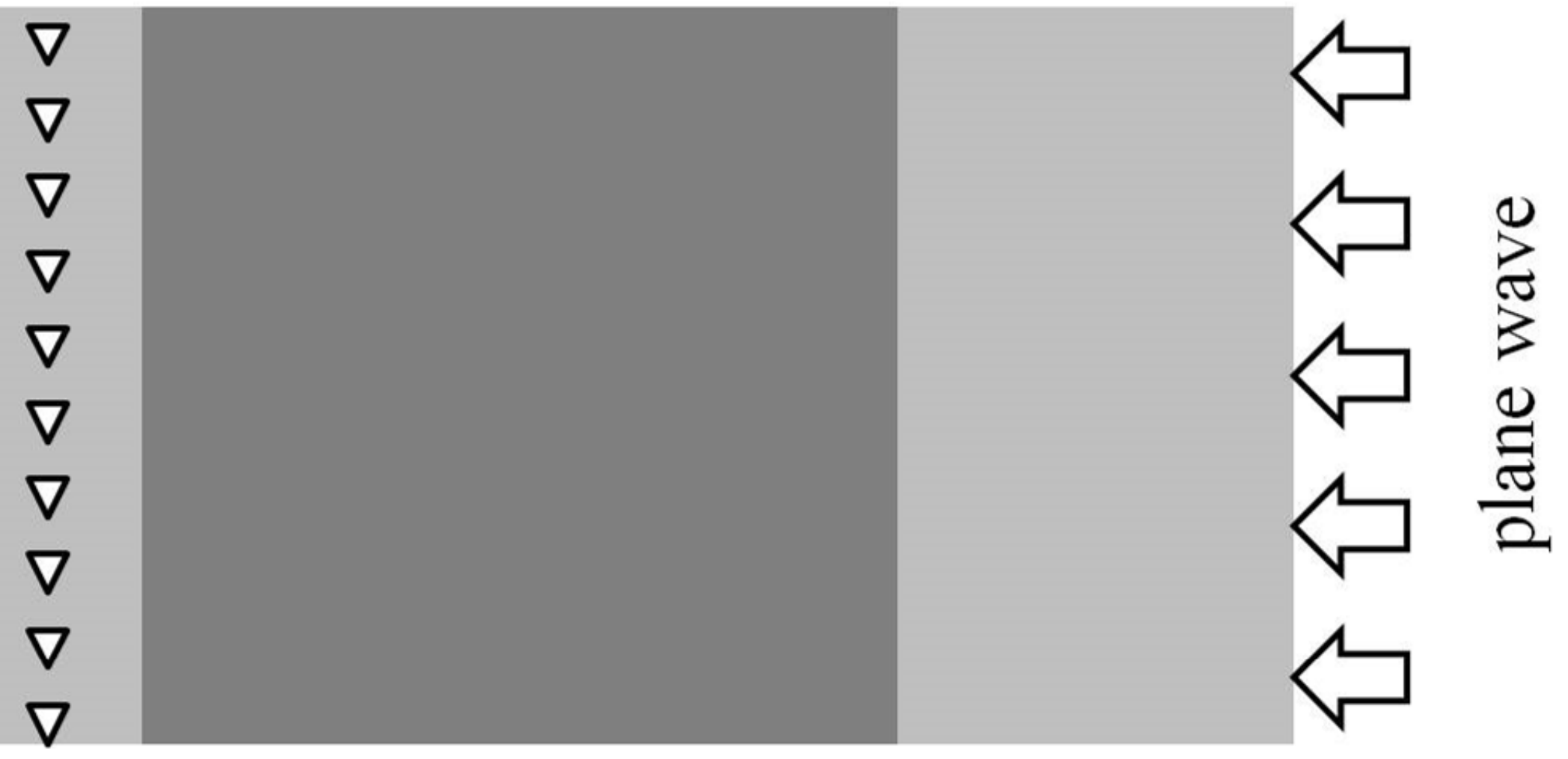

Fig. 6 
(a)

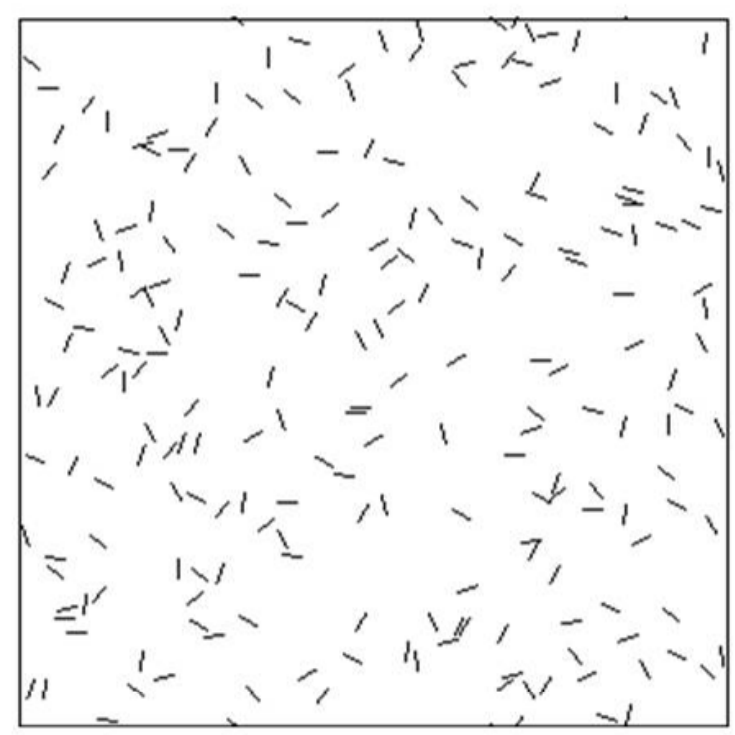

(b)

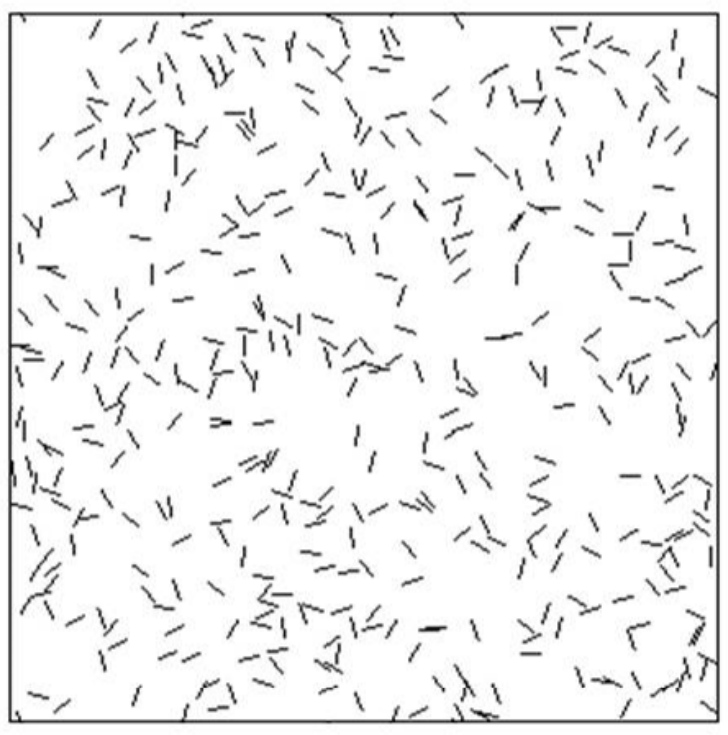

(c)

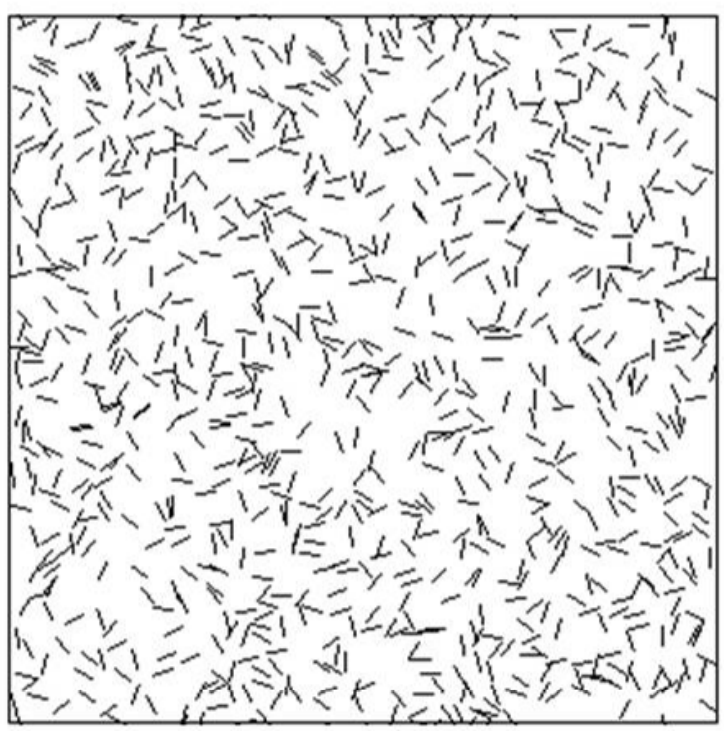

Fig. 7 


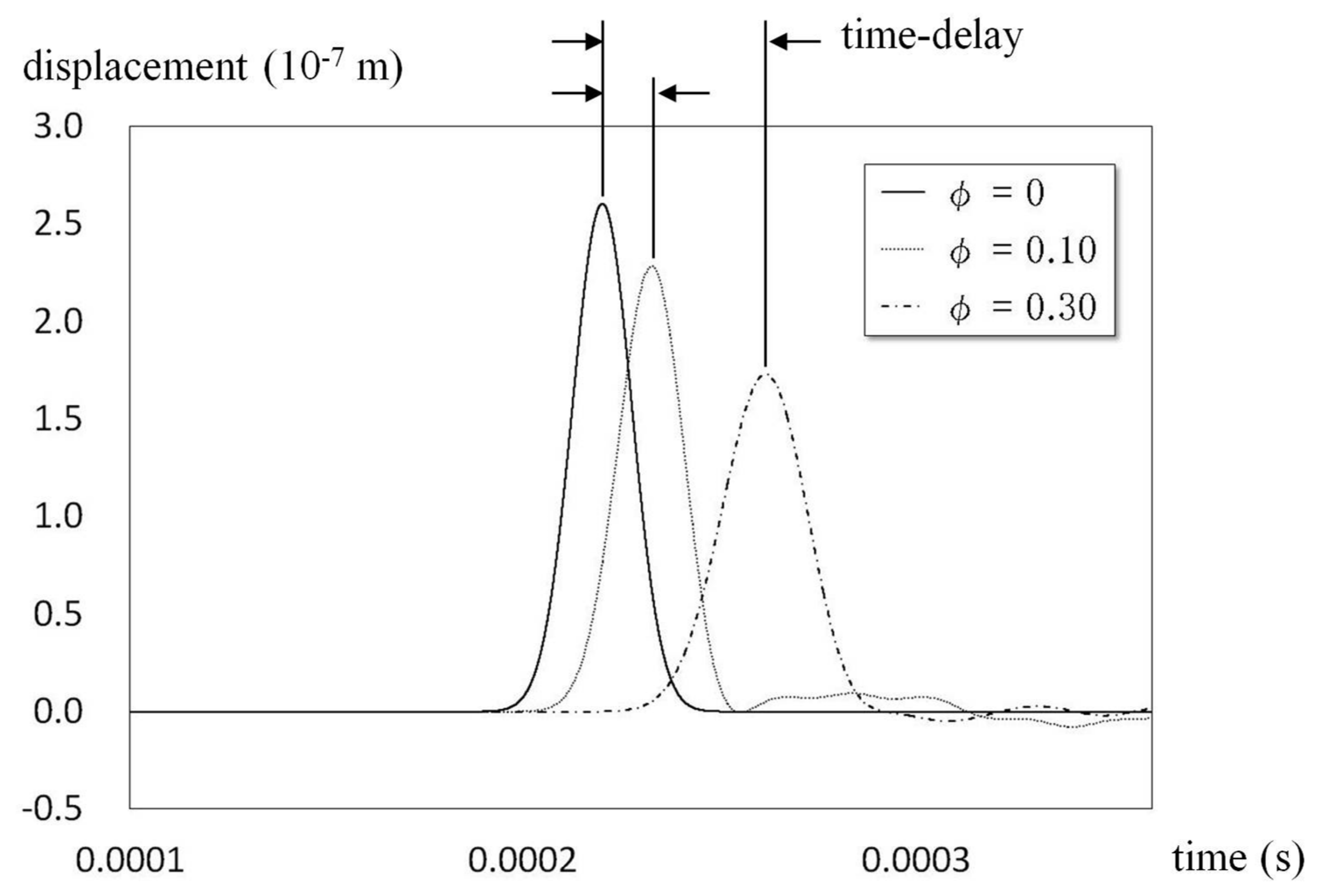

Fig. 8 


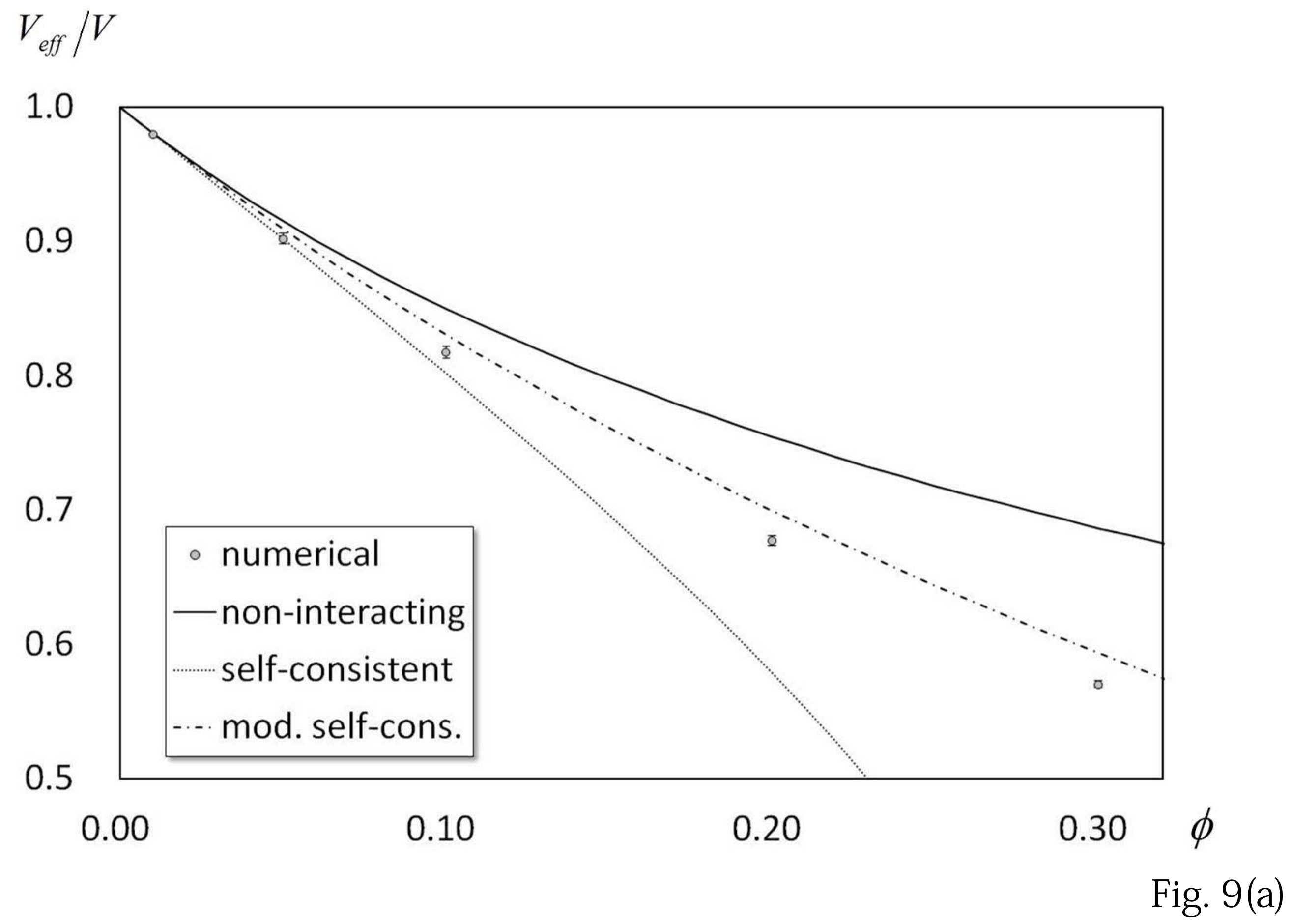




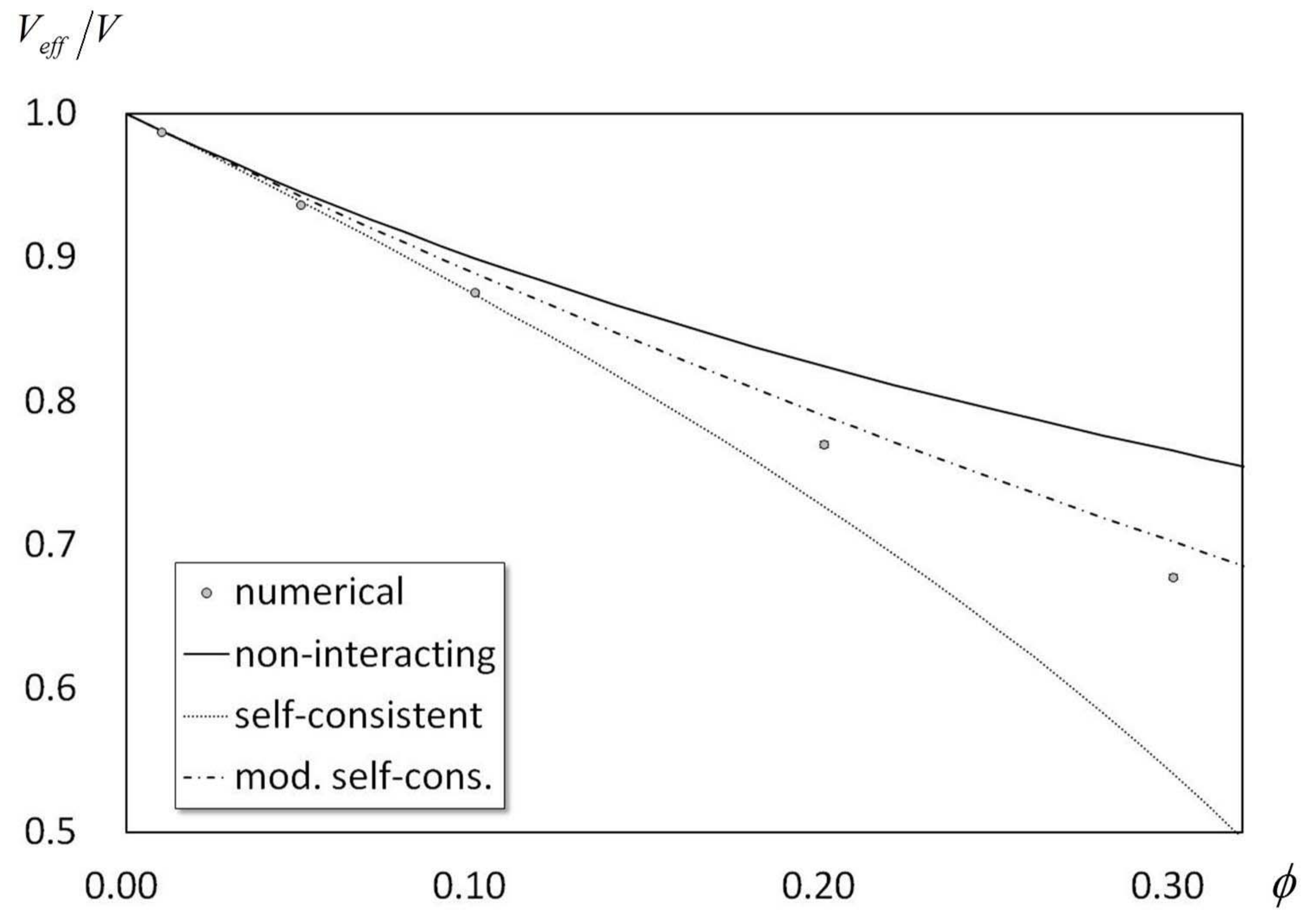

Fig. 9(b) 
Fig. 10(a)

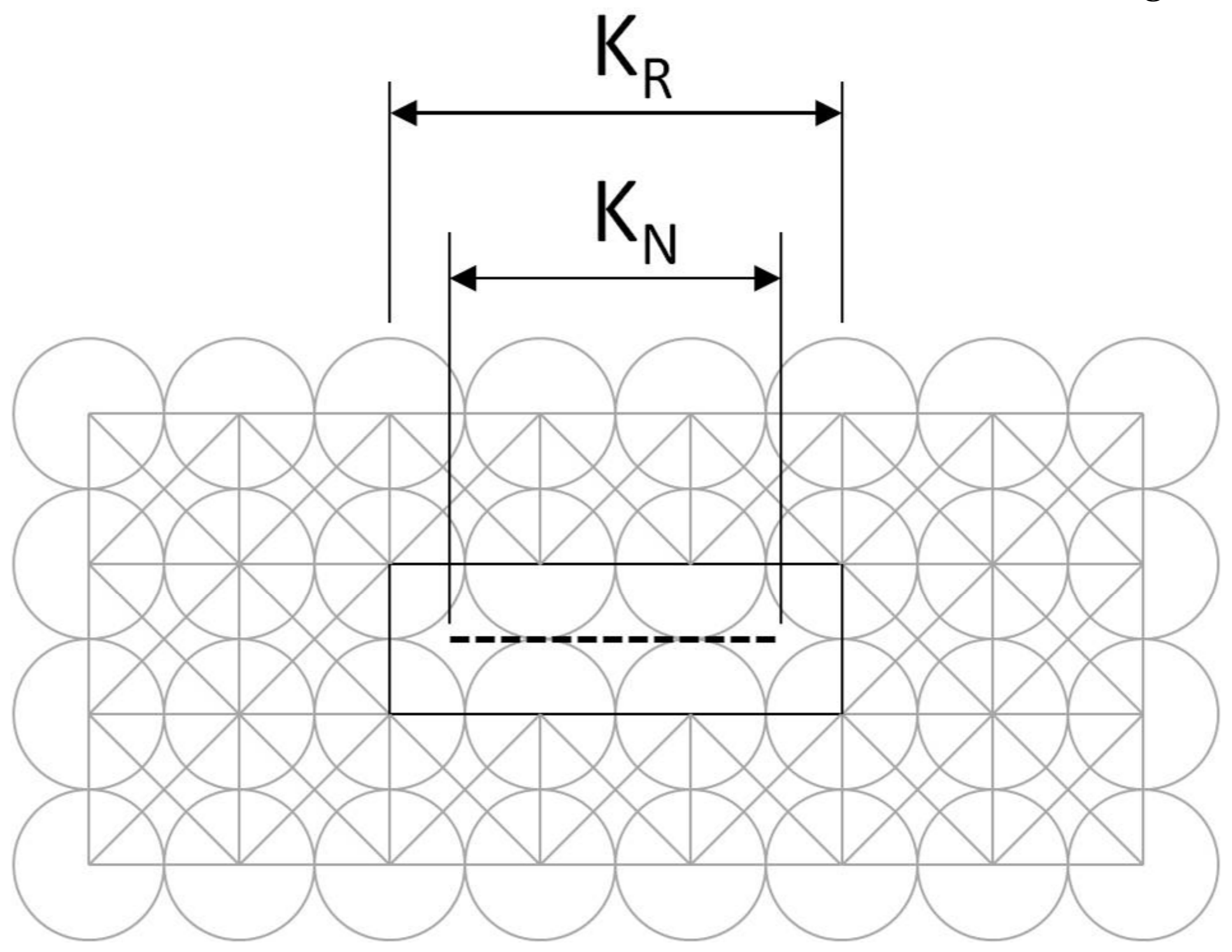


Fig. 10(b)

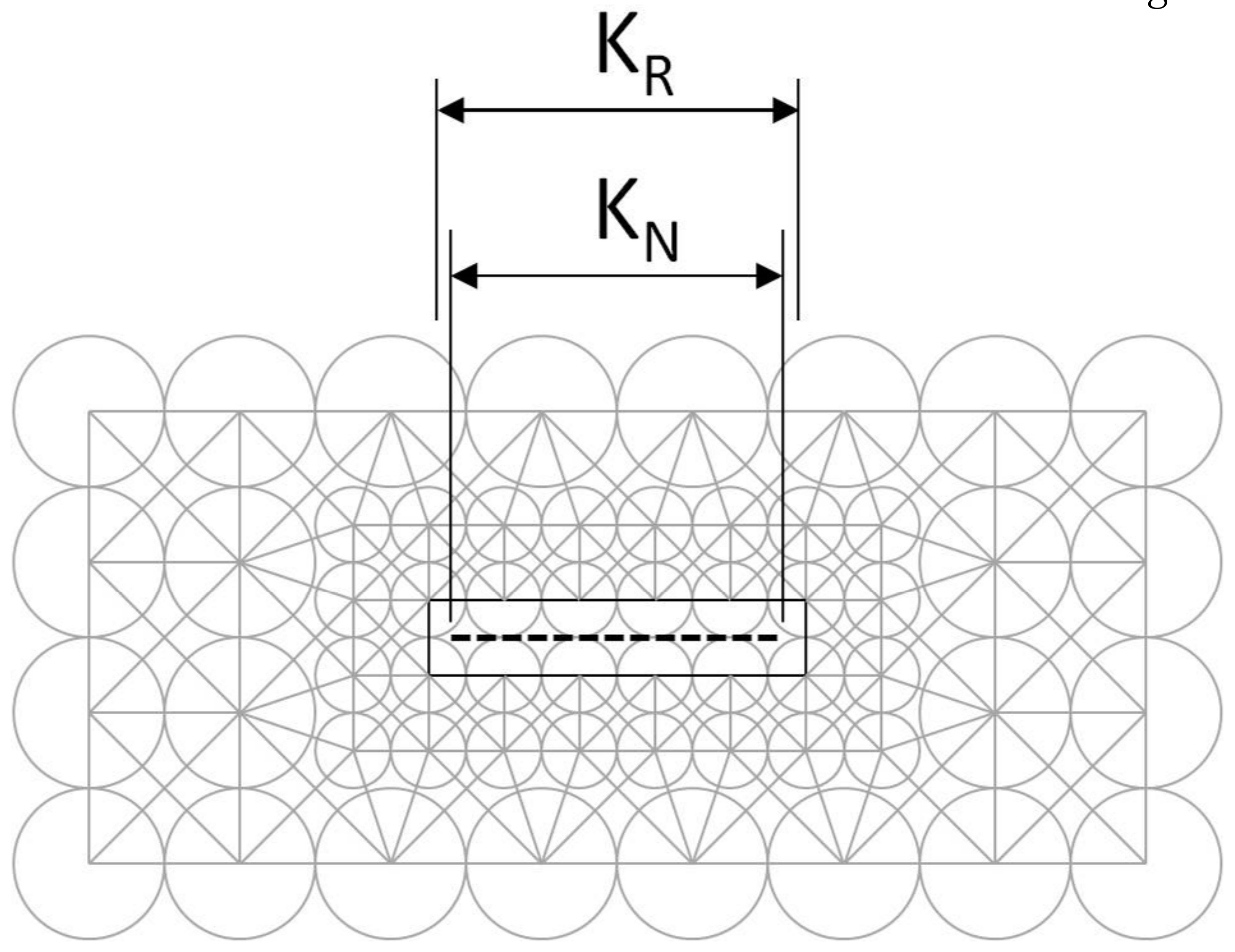




\section{cracked region}
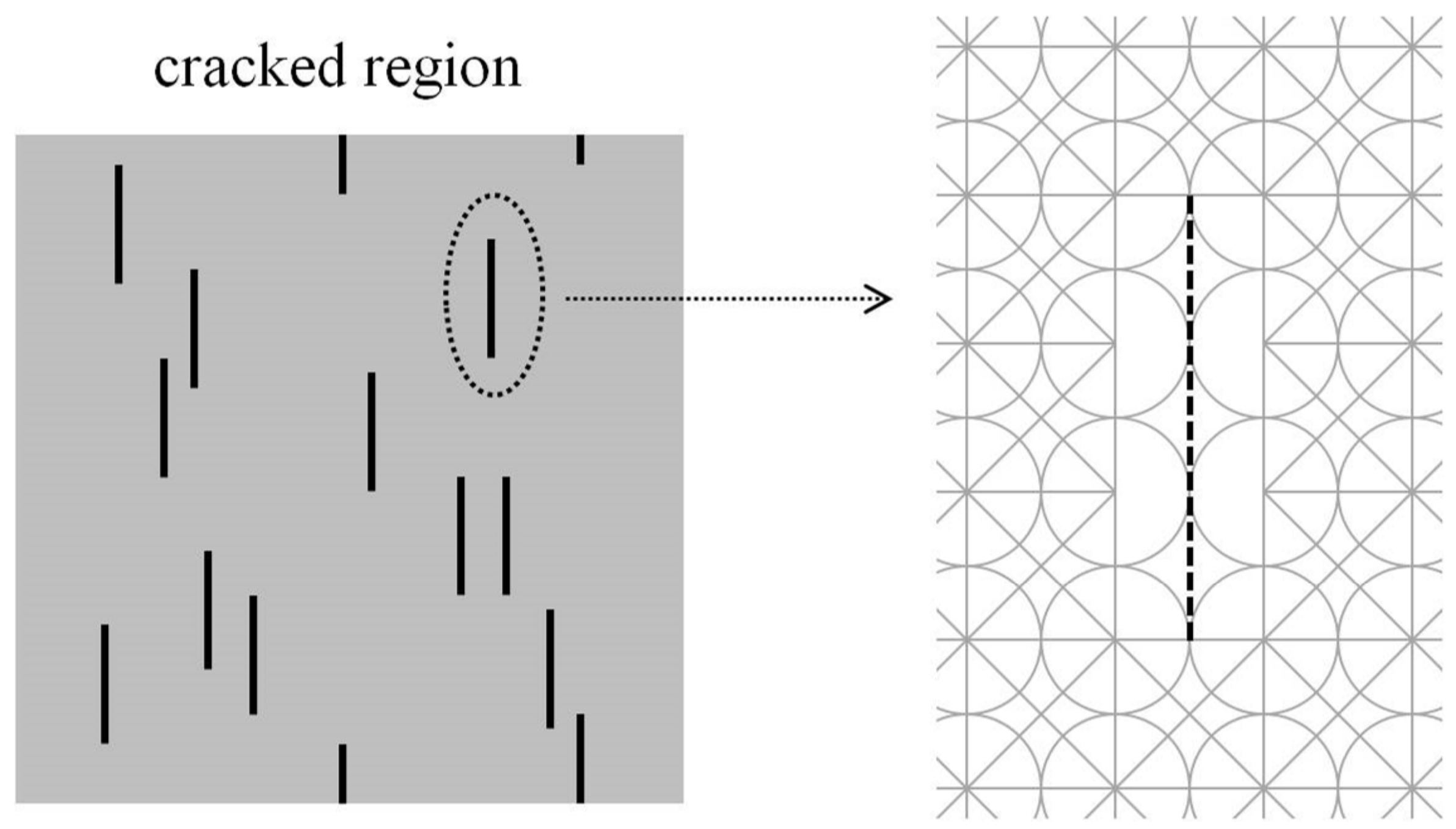

Fig. 11 


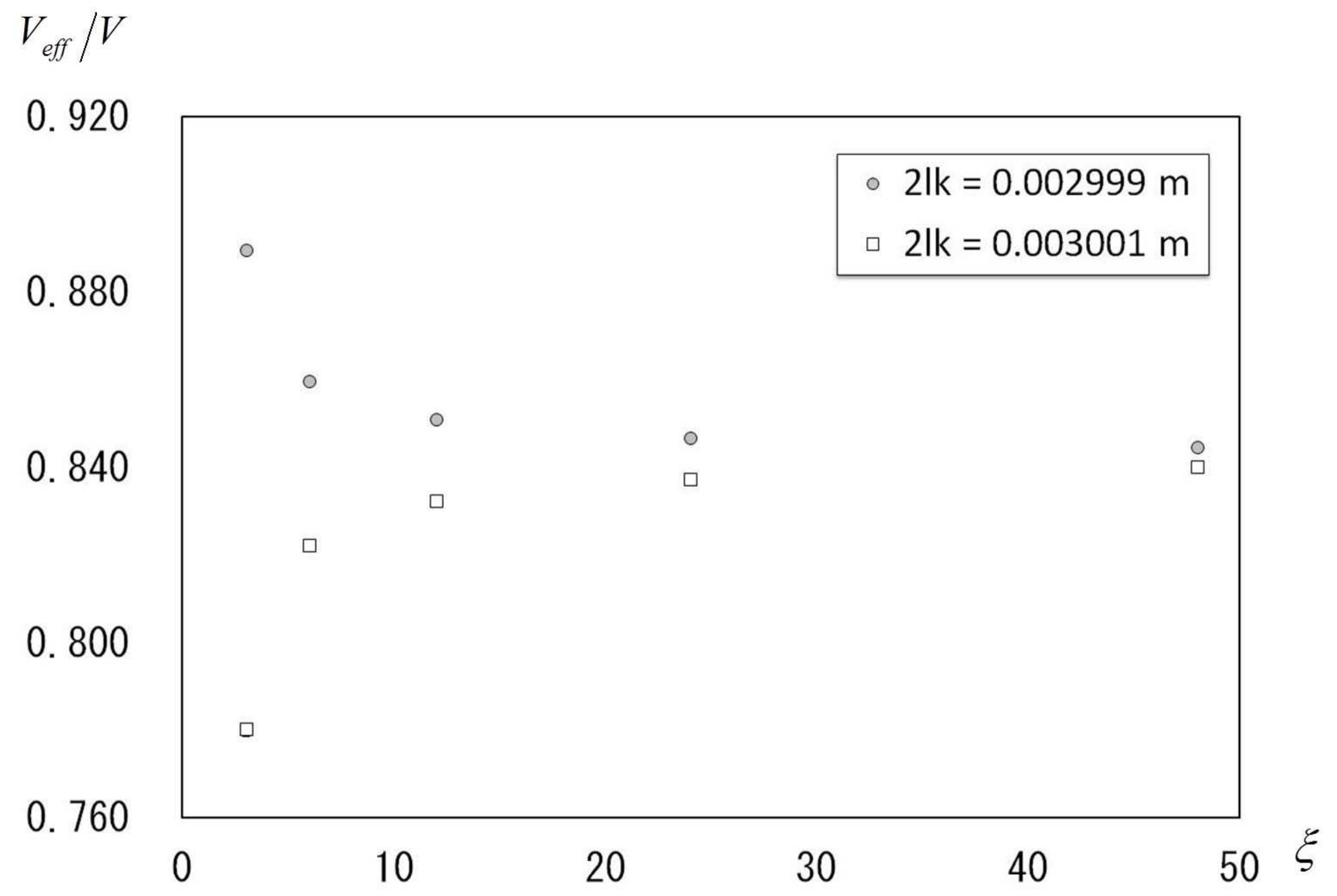

Fig. 12 


\section{(a)}

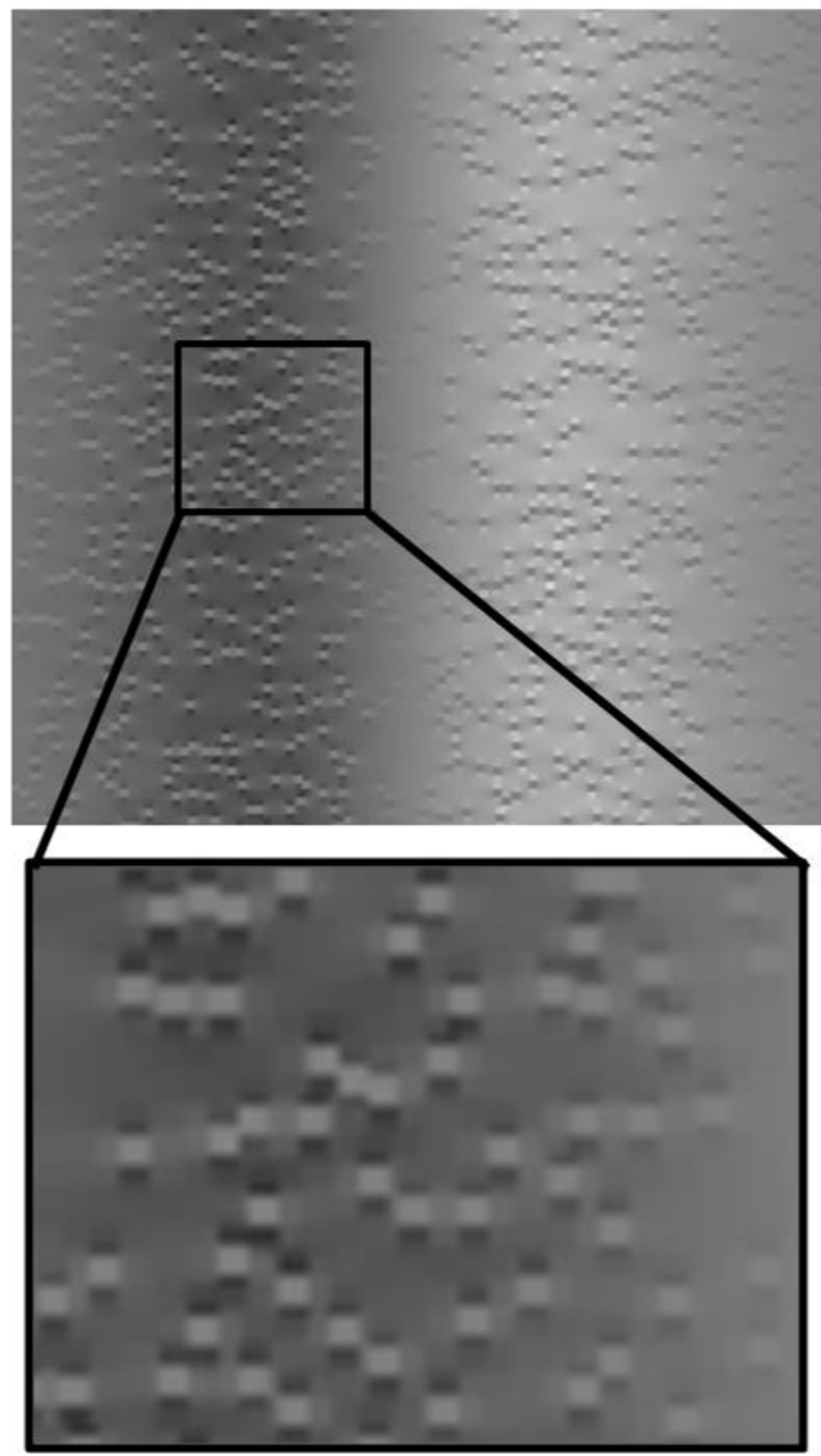

Fig. 13(a) 
(b)

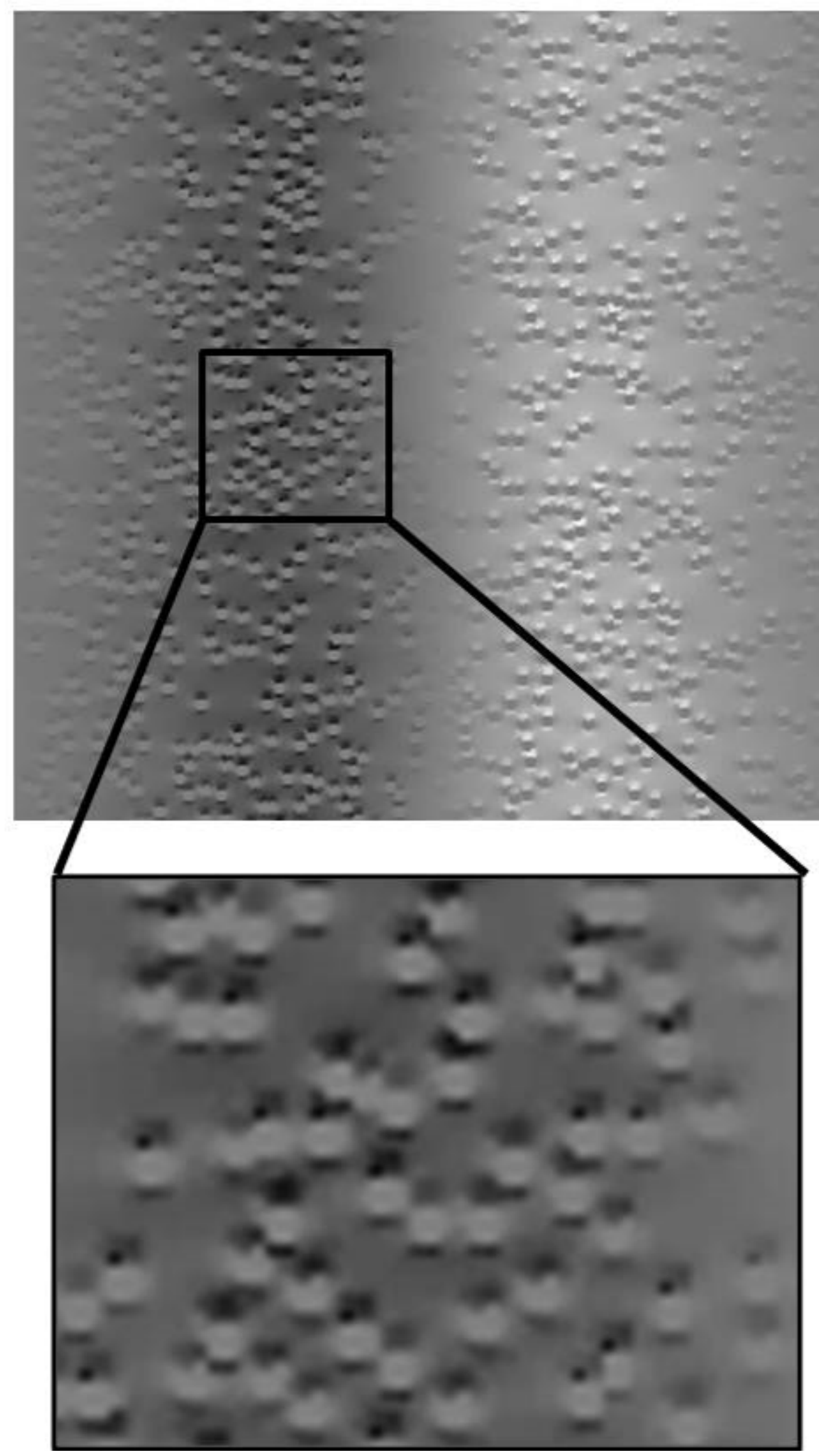

Fig. 13(b) 


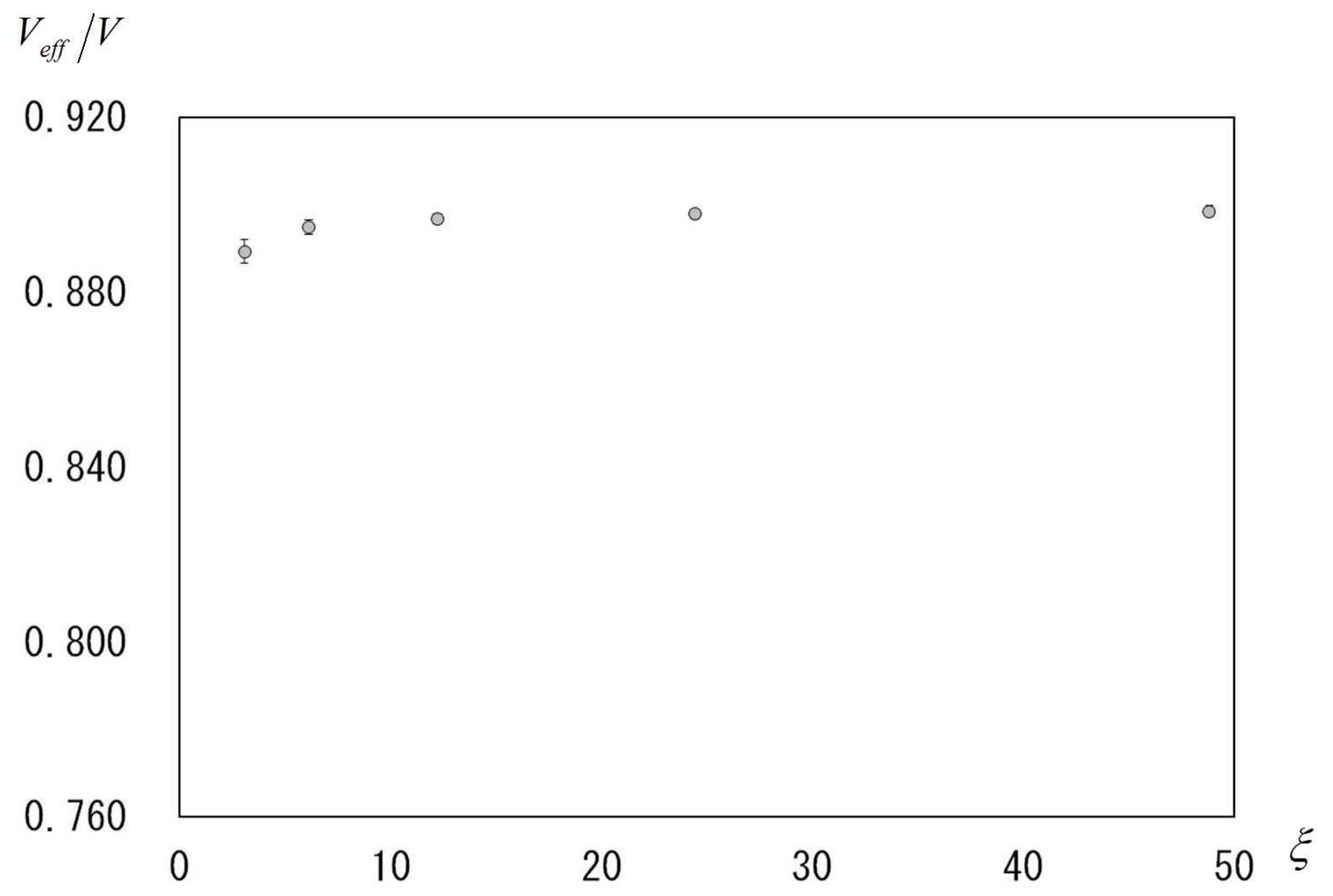

Fig. 14 